\title{
Documenting decapod biodiversity in the
}

\section{Caribbean from DNA barcodes generated during field training in taxonomy}

\author{
Dagoberto E. Venera-Pontón ${ }^{\ddagger} \S$, Amy C. Driskelll, Sammy De Graveף, Darryl L. Felder§, \\ Justin A. Scioli§, Rachel Collin $¥$ \\ ‡ Smithsonian Tropical Research Institute, Balboa, Panama \\ § University of Louisiana at Lafayette, Lafayette, United States of America \\ | Laboratories of Analytical Biology, Department of Invertebrate Zoology, Smithsonian Institution, Washington, D.C., United \\ States of America \\ I Oxford University Museum of Natural History, Oxford, United Kingdom
}

\section{Corresponding author: Dagoberto E. Venera-Pontón (dagovenera@gmail.com)}

Academic editor: John James Wilson

Received: 15 Oct 2019 | Accepted: 28 Dec 2019 | Published: 07 Jan 2020

Citation: Venera-Pontón DE, Driskell AC, De Grave S, Felder DL, Scioli JA, Collin R (2020) Documenting decapod biodiversity in the Caribbean from DNA barcodes generated during field training in taxonomy. Biodiversity Data Journal 8: e47333. https://doi.org/10.3897/BDJ.8.e47333

\begin{abstract}
DNA barcoding is a useful tool to identify the components of mixed or bulk samples, as well as to determine individuals that lack morphologically diagnostic features. However, the reference database of DNA barcode sequences is particularly sparsely populated for marine invertebrates and for tropical taxa. We used samples collected as part of two field courses, focused on graduate training in taxonomy and systematics, to generate DNA sequences of the barcode fragments of cytochrome $c$ oxidase subunit I (COI) and mitochondrial ribosomal $16 \mathrm{~S}$ genes for 447 individuals, representing at least 129 morphospecies of decapod crustaceans. COI sequences for $36 \%(51 / 140)$ of the species and $16 \mathrm{~S}$ sequences for $26 \%(37 / 140)$ of the species were new to GenBank. Automatic Barcode Gap Discovery identified 140 operational taxonomic units (OTUs) which largely coincided with the morphospecies delimitations. Barcode identifications (i.e. matches to identified sequences) were especially useful for OTUs within Synalpheus, a group that is notoriously difficult to identify and rife with cryptic species, a number of which we could not
\end{abstract}


identify to species, based on morphology. Non-concordance between morphospecies and barcode OTUs also occurred in a few cases of suspected cryptic species. As mitochondrial pseudogenes are particularly common in decapods, we investigate the potential for this dataset to include pseudogenes and discuss the utility of these sequences as species identifiers (i.e. barcodes). These results demonstrate that material collected and identified during training activities can provide useful incidental barcode reference samples for under-studied taxa.

\section{Keywords}

cytochrome c oxidase I, Panama, Bocas del Toro, shrimps, crabs

\section{Introduction}

A shortage of taxonomic expertise is one of the current challenges facing those engaged in identifying, classifying, utilising and conserving the world's biodiversity (Giangrande 2003, Vernooy et al. 2010, Cardoso et al. 2011, Ebach et al. 2011, Wägele et al. 2011, Sluys 2013). The recent application of DNA techniques, the compilation of large taxonomic databases (Costello et al. 2013) and the use of bioinformatics approaches like GIS, have rejuvenated interest in taxonomic data. Unfortunately, this increase in relevance and interest has been counteracted by the gradual loss of integrative taxonomic expertise (Drew 2011, Coleman 2015). This recent decline has limited attempts to document the world's biodiversity and limits the rate at which high-profile initiatives, such as the Census for Marine Life, Barcode of Life (BOLD; Ratnasingham and Hebert 2007) and WoRMS, can generate, agglomerate and synthesise biodiversity knowledge. There is a particular need for new experts specialising in marine biodiversity, where it is estimated that $30-60 \%$ (Appeltans et al. 2012) or even 90\% (Mora et al. 2011) of eukaryotic species remain to be described or discovered. There is also a particular shortage of taxonomists and data from developing countries and countries with economies in transition.

DNA barcoding is a useful tool for the identification of samples that cannot be identified, based on traditional morphological methods (Bucklin et al. 2011, Bucklin et al. 2016, Geiger et al. 2016). Short, easily amplifiable fragments that vary amongst closely related species are sequenced from specimens identified by experts and then used as a reference set to compare with sequences from unidentified or unidentifiable samples (Hebert and Gregory 2005, Hajibabaei et al. 2007, Bucklin et al. 2011, Bucklin et al. 2016, Geiger et al. 2016). For animals, the most widely used barcode is cytochrome c oxidase subunit I (COI; Hebert et al. 2003), followed by the $16 \mathrm{~S}$ large subunit ribosomal RNA (16S), another mitochondrial marker (see Mantelatto et al. 2017, Collin et al. 2018, Collin et al. 2019, Morín et al. 2019). This approach is useful in a variety of contexts, including identifying components of gut contents and bulk environmental samples. However, the global DNA barcode database (BOLD; Ratnasingham and Hebert 2007) is still sparsely populated, specifically for invertebrates and especially for tropical taxa. In many cases, even common, relatively easily identified and well-known taxa do not yet have sequences of the DNA 
barcode fragment of $\mathrm{COI}$ publicly available in GenBank. For example, Raupach and Radulovici (2015) showed a particular lack of DNA barcode studies for crustaceans from the Caribbean, amongst other regions. Therefore, activities that can aid in generating DNA reference barcodes for commonly encountered species, even without a comprehensive effort to exhaustively document species in a particular group or fauna, can make a significant contribution to the utility of the barcode database. In addition, improved taxonomic coverage may assist in narrowing down the possible identities of unknown samples that do not match any reference sample.

Here, we used material collected as part of graduate taxonomy training workshops in Bocas del Toro, Panama to generate a reference set of DNA barcodes of common shallowwater decapods of the Caribbean coast of Panama. Decapods are one invertebrate group that, despite its importance and high diversity, still has low DNA barcode coverage in the tropics (Raupach and Radulovici 2015). Our hope was that by combining the two, not only could trainees become familiar with processing material for subsequent DNA extraction, but that the contribution of biodiversity data to global databases would help garner continued support and help make training activities more sustainable (Cancian de Araujo et al. 2018). Due to the wide geographic ranges of many taxa throughout the Caribbean and the potential for gene flow between Bocas del Toro and other parts of the region via dispersal of planktonic larvae on ocean currents (Cowen et al. 2007, Cowen and Sponaugle 2009, Schill et al. 2015), our barcode library may be useful in other zones of the Caribbean Sea.

\section{Material and methods}

\section{Collection}

Specimens for DNA barcoding were collected during two workshops of the Training in Tropical Taxonomy programme run by the Smithsonian Tropical Research Institute in Bocas del Toro, Panama. The "Shrimp Taxonomy (Caridea, Dendrobranchiata and Stenopodidea)" course in 2008 included 13 students from eight countries (Mexico, UK, US, Colombia, Slovenia, Brazil, Australia and Costa Rica) and the "Taxonomy and Biology of Decapod Crustaceans" course included 13 students from five countries (US, Colombia, Brazil, Argentina and Costa Rica) in 2011. These 2-week workshops, each led by one of us (SDG and DF, respectively) and co-instructed by A. Anker and F. Mantelatto, respectively, were aimed at graduate student training but also included undergraduate students and post-doctoral professionals seeking training in systematics and identification of the focal groups. During two weeks, students collected and identified specimens as part of their training and, when the animals were intact and well-enough preserved to make useful vouchers, tissue samples were taken by us for DNA barcoding (see section on DNA sequencing). Therefore, unlike other studies that included training sessions in DNA barcoding (e.g. Harris and Bellino 2013), our study simply made use of specimens collected and identified under the supervision of taxonomic experts during taxonomy training workshops. All specimens were collected from Bahia Almirante, especially from 
sites in and around Isla Colon and Isla Bastimentos. No documentation of sampling effort was made, as collections were opportunistic and arranged around the other activities of the courses. The marine invertebrate diversity of this area has been documented in some detail (e.g. Collin et al. 2005, Rocha et al. 2005, Bonnet and Rocha 2011, Goodheart et al. 2016). A detailed checklist is available for shrimps (De Grave and Anker 2017), but no checklists or comprehensive surveys are available for brachyurans or anomurans.

Vouchers from the decapod course are deposited in the decapod collection of the University of Louisiana at Lafayette (ULL) which is currently being transferred to the Smithsonian Natural History Museum (USNM). Reference numbers from the UL collection are provided in the dataset associated to this research (dx.doi.org/10.5883/DSCRUSTACE and Table 1). Many vouchers from the shrimp course are currently stored in the Zoological Collection of the Oxford University Museum of Natural History (OUMNH.ZC see Table 1). However, a number of shrimp samples were transferred to the Museu Nacional de Brazil and were subsequently lost in the fire that destroyed the museum in 2018. This lost material is listed without voucher numbers (Table 1). Additionally, a few shrimp samples are stored in the Natural History Museum of Vienna (NHMW).

Table 1.

Summary of the Operational Taxonomic Units (OTUs) detected in this study, their morphological identification, Barcode Index Numbers (BINs) in BOLD and their contribution to information in GenBank. Whenever our OTU provided a new species name in GenBank, the morphospecies was tagged with the symbols ${ }^{\wedge}$ or $\sim$ if the contribution occurred in COI or $16 \mathrm{~S}$, respectively. New BINs generated in this study are indicated with asterisks*.

\begin{tabular}{|c|c|c|c|c|c|c|}
\hline Family & Morphospecies & Museum ID & BINs & \#Inds & $\begin{array}{l}\text { COI new } \\
\text { to } \\
\text { GenBank } \\
(<95 \% \\
\text { identity) }\end{array}$ & $\begin{array}{l}16 S \text { new } \\
\text { to } \\
\text { GenBank } \\
(<97 \% \\
\text { identity) }\end{array}$ \\
\hline \multicolumn{7}{|c|}{ Porcellanid Crabs: Anomura } \\
\hline Albuneidae & $\begin{array}{l}\text { Lepidopa cf. } \\
\text { richmondi }\end{array}$ & ULLZ13327 & BOLD:ACT9781* & 1 & NEW & NEW \\
\hline Diogenidae & Calcinus tibicen & $\begin{array}{l}\text { ULLZ13427, } \\
\text { ULLZ13444, } \\
\text { ULLZ13426, } \\
\text { ULLZ13445 }\end{array}$ & BOLD:AAE8392 & 4 & - & - \\
\hline Diogenidae & $\begin{array}{l}\text { Clibanarius } \\
\text { antillensis }\end{array}$ & $\begin{array}{l}\text { ULLZ13597, } \\
\text { ULLZ13599, } \\
\text { ULLZ13619, } \\
\text { ULLZ13617, } \\
\text { ULLZ13618 }\end{array}$ & BOLD:AAK1039 & 5 & - & - \\
\hline
\end{tabular}




\begin{tabular}{|c|c|c|c|c|c|c|}
\hline Family & Morphospecies & Museum ID & BINs & \#lnds & $\begin{array}{l}\text { COI new } \\
\text { to } \\
\text { GenBank } \\
\text { (<95\% } \\
\text { identity) }\end{array}$ & $\begin{array}{l}16 \mathrm{~S} \text { new } \\
\text { to } \\
\text { GenBank } \\
\text { (<97\% } \\
\text { identity) }\end{array}$ \\
\hline Diogenidae & $\begin{array}{l}\text { Clibanarius } \\
\text { sclopetarius }\end{array}$ & ULLZ13353 & BOLD:ACB6405 & 1 & - & - \\
\hline Diogenidae & Dardanus fucosus ${ }^{\wedge}$ & $\begin{array}{l}\text { ULLZ13352, } \\
\text { ULLZ13602 }\end{array}$ & BOLD:AAI5823 & 2 & NEW & NEW \\
\hline Diogenidae & $\begin{array}{l}\text { Paguristes } \\
\text { tortugae }^{\wedge}\end{array}$ & $\begin{array}{l}\text { ULLZ13663, } \\
\text { ULLZ13664, } \\
\text { ULLZ13665, } \\
\text { ULLZ13707, } \\
\text { ULLZ13708, } \\
\text { ULLZ13709 }\end{array}$ & BOLD:ACT9547* & 6 & - & NEW \\
\hline Hippidae & Emerita sp. & $\begin{array}{l}\text { ULLZ13325, } \\
\text { ULLZ13456, } \\
\text { ULLZ13457, } \\
\text { ULLZ13690 }\end{array}$ & BOLD:ACU0009 & 4 & - & - \\
\hline Hippidae & $\begin{array}{l}\text { Hippa testudinaria } \\
\wedge \sim\end{array}$ & $\begin{array}{l}\text { ULLZ13326, } \\
\text { ULLZ13706, } \\
\text { ULLZ13613 }\end{array}$ & BOLD:ACT9780* & 3 & NEW & NEW \\
\hline Paguridae & $\begin{array}{l}\text { Pagurus } \\
\text { brevidactylus }\end{array}$ & ULLZ13660 & BOLD:AAF9919 & 1 & NEW & NEW \\
\hline Paguridae & $\begin{array}{l}\text { Pagurus } \\
\text { criniticornis }\end{array}$ & ULLZ13635 & BOLD:ACU0173* & 1 & NEW & NEW \\
\hline Paguridae & $\begin{array}{l}\text { Pagurus } \\
\text { criniticornis, P. nr. } \\
\text { criniticornis }\end{array}$ & $\begin{array}{l}\text { ULLZ13482, } \\
\text { ULLZ13483a, } \\
\text { ULLZ13483b, } \\
\text { ULLZ13633, } \\
\text { ULLZ13634, } \\
\text { ULLZ13692, } \\
\text { ULLZ13693, } \\
\text { ULLZ13714, } \\
\text { ULLZ13632 }\end{array}$ & BOLD:ACT9783* & 9 & NEW & NEW \\
\hline Paguridae & $\begin{array}{l}\text { Pagurus } n r . \\
\text { maclaughlinae }\end{array}$ & $\begin{array}{l}\text { ULLZ13384, } \\
\text { ULLZ13385 }\end{array}$ & BOLD:ACT9865 & 2 & NEW & NEW \\
\hline Porcellanidae & $\begin{array}{l}\text { Megalobrachium } \\
\text { roseum }\end{array}$ & $\begin{array}{l}\text { ULLZ13449, } \\
\text { ULLZ13450 }\end{array}$ & BOLD:ACT9407 & 2 & - & - \\
\hline
\end{tabular}




\begin{tabular}{|c|c|c|c|c|c|c|}
\hline Family & Morphospecies & Museum ID & BINs & \#Inds & $\begin{array}{l}\text { COI new } \\
\text { to } \\
\text { GenBank } \\
\text { (<95\% } \\
\text { identity) }\end{array}$ & $\begin{array}{l}16 \mathrm{~S} \text { new } \\
\text { to } \\
\text { GenBank } \\
\text { (<97\% } \\
\text { identity) }\end{array}$ \\
\hline Porcellanidae & $\begin{array}{l}\text { Pachycheles } \\
\text { chacei^ }^{\wedge}\end{array}$ & $\begin{array}{l}\text { ULLZ13436, } \\
\text { ULLZ13437, } \\
\text { ULLZ13438 }\end{array}$ & BOLD:ACT9930* & 3 & NEW & - \\
\hline Porcellanidae & $\begin{array}{l}\text { Pachycheles } \\
\text { cristobalensis^ } ~\end{array}$ & $\begin{array}{l}\text { ULLZ13421, } \\
\text { ULLZ13428, } \\
\text { ULLZ13429, } \\
\text { ULLZ13430 }\end{array}$ & BOLD:ACU0707* & 4 & NEW & - \\
\hline Porcellanidae & $\begin{array}{l}\text { Pachycheles } \\
\text { tuerkayi^ }^{\wedge}\end{array}$ & $\begin{array}{l}\text { ULLZ13354, } \\
\text { ULLZ13586, } \\
\text { ULLZ13587, } \\
\text { ULLZ13593, } \\
\text { ULLZ13595, } \\
\text { ULLZ13701 }\end{array}$ & BOLD:ACU0397* & 6 & NEW & - \\
\hline Porcellanidae & $\begin{array}{l}\text { Petrolisthes } \\
\text { armatus }\end{array}$ & $\begin{array}{l}\text { ULLZ13315, } \\
\text { ULLZ13369, } \\
\text { ULLZ13370, } \\
\text { ULLZ13371, } \\
\text { ULLZ13433, } \\
\text { ULLZ13434 }\end{array}$ & BOLD:AAA2699 & 6 & - & - \\
\hline Porcellanidae & $\begin{array}{l}\text { Petrolisthes } \\
\text { galathinus }\end{array}$ & $\begin{array}{l}\text { ULLZ13339, } \\
\text { ULLZ13454, } \\
\text { ULLZ13612, } \\
\text { ULLZ13672, } \\
\text { ULLZ13673, } \\
\text { ULLZ13674 }\end{array}$ & BOLD:ACG7994 & 6 & - & - \\
\hline Porcellanidae & $\begin{array}{l}\text { Petrolisthes } \\
\text { jugosus }\end{array}$ & $\begin{array}{l}\text { ULLZ13423, } \\
\text { ULLZ13424 }\end{array}$ & BOLD:ACU0611* & 2 & NEW & NEW \\
\hline \multicolumn{7}{|c|}{ Brachyuran Crabs: Brachyura } \\
\hline Calappidae & Calappa galloides & ULLZ13575 & BOLD:AAV0354 & 1 & - & - \\
\hline Calappidae & Calappa ocellata & ULLZ13404 & BOLD:ACT9710 & 1 & - & - \\
\hline Epialtidae & $\begin{array}{l}\text { Acanthonyx } \\
\text { petiverii }\end{array}$ & $\begin{array}{l}\text { ULLZ13398, } \\
\text { ULLZ13399, } \\
\text { ULLZ13422 }\end{array}$ & BOLD:ACG7625 & 3 & - & - \\
\hline
\end{tabular}




\begin{tabular}{|c|c|c|c|c|c|c|}
\hline Family & Morphospecies & Museum ID & BINs & \#lnds & $\begin{array}{l}\text { COI new } \\
\text { to } \\
\text { GenBank } \\
\text { (<95\% } \\
\text { identity) }\end{array}$ & $\begin{array}{l}16 S \text { new } \\
\text { to } \\
\text { GenBank } \\
\text { (<97\% } \\
\text { identity) }\end{array}$ \\
\hline Epialtidae & $\begin{array}{l}\text { Epialtus } \\
\text { bituberculatus }\end{array}$ & $\begin{array}{l}\text { ULLZ13572, } \\
\text { ULLZ13573, } \\
\text { ULLZ13574, } \\
\text { ULLZ13603, } \\
\text { ULLZ13601, } \\
\text { ULLZ13604 }\end{array}$ & BOLD:ACG7953 & 6 & - & - \\
\hline Epialtidae & $\begin{array}{l}\text { Macrocoeloma } \\
\text { diplacanthum^ }\end{array}$ & ULLZ13313 & BOLD:ACT8809* & 1 & NEW & NEW \\
\hline Epialtidae & $\begin{array}{l}\text { Macrocoeloma } \\
\text { subparellelum } \sim\end{array}$ & ULLZ13311 & BOLD:ACT9697* & 1 & NEW & NEW \\
\hline Epialtidae & $\begin{array}{l}\text { Macrocoeloma } \\
\text { trispinosum }\end{array}$ & $\begin{array}{l}\text { ULLZ13374, } \\
\text { ULLZ13375, } \\
\text { ULLZ13455, } \\
\text { ULLZ13667 }\end{array}$ & BOLD:ACG7680 & 4 & - & - \\
\hline Epialtidae & Pitho mirabilis & ULLZ13592 & BOLD:ACT8761* & 1 & NEW & NEW \\
\hline Eriphiidae & Eriphia gonagra & $\begin{array}{l}\text { ULLZ13395, } \\
\text { ULLZ13396, } \\
\text { ULLZ13397 }\end{array}$ & BOLD:ACG8098 & 3 & - & - \\
\hline Gecarcinidae & $\begin{array}{l}\text { Cardisoma } \\
\text { guanhumi }\end{array}$ & ULLZ13341 & BOLD:ACT8737 & 1 & - & - \\
\hline Grapsidae & $\begin{array}{l}\text { Goniopsis } \\
\text { cruentata }\end{array}$ & ULLZ13661 & BOLD:ACG7928 & 1 & - & - \\
\hline Grapsidae & Grapsus grapsus ${ }^{\wedge}$ & $\begin{array}{l}\text { ULLZ13414, } \\
\text { ULLZ13415, } \\
\text { ULLZ13416 }\end{array}$ & BOLD:ACU0001* & 3 & - & - \\
\hline Grapsidae & $\begin{array}{l}\text { Pachygrapsus } \\
\text { gracilis }\end{array}$ & $\begin{array}{l}\text { ULLZ13358, } \\
\text { ULLZ13656 }\end{array}$ & BOLD:ACU0366 & 2 & - & - \\
\hline Grapsidae & $\begin{array}{l}\text { Pachygrapsus } \\
\text { transversus }\end{array}$ & $\begin{array}{l}\text { ULLZ13606, } \\
\text { ULLZ13607, } \\
\text { ULLZ13608 }\end{array}$ & BOLD:AAG9839 & 3 & - & - \\
\hline Inachidae & $\begin{array}{l}\text { Stenorhynchus } \\
\text { seticornis }\end{array}$ & $\begin{array}{l}\text { ULLZ13359, } \\
\text { ULLZ13360, } \\
\text { ULLZ13400 }\end{array}$ & BOLD:AAJ5290 & 3 & - & - \\
\hline Inachoididae & Inachoides sp. & ULLZ13463 & BOLD:ACT9182* & 1 & NEW & - \\
\hline
\end{tabular}




\begin{tabular}{|c|c|c|c|c|c|c|}
\hline Family & Morphospecies & Museum ID & BINs & \#Inds & $\begin{array}{l}\text { COI new } \\
\text { to } \\
\text { GenBank } \\
\text { (<95\% } \\
\text { identity) }\end{array}$ & $\begin{array}{l}16 S \text { new } \\
\text { to } \\
\text { GenBank } \\
\text { (<97\% } \\
\text { identity) }\end{array}$ \\
\hline Leucosiidae & $\begin{array}{l}\text { Speloeophorus } \\
\text { nodosus }^{\wedge}\end{array}$ & ULLZ13317 & BOLD:ACT8927* & 1 & NEW & - \\
\hline Majidae & Thoe puella & $\begin{array}{l}\text { ULLZ13582, } \\
\text { ULLZ13583 }\end{array}$ & BOLD:ACU0496* & 2 & - & - \\
\hline Menippidae & Menippe nodifrons & $\begin{array}{l}\text { ULLZ13481, } \\
\text { ULLZ13654, } \\
\text { ULLZ13655 }\end{array}$ & BOLD:AAX4629 & 3 & - & - \\
\hline Mithracidae & $\begin{array}{l}\text { Amphithrax } \\
\text { aculeatus }\end{array}$ & ULLZ13596 & BOLD:ACU0682 & 1 & - & - \\
\hline Mithracidae & $\begin{array}{l}\text { Mithraculus } \\
\text { cinctimanus }\end{array}$ & $\begin{array}{l}\text { ULLZ13328, } \\
\text { ULLZ13408, } \\
\text { ULLZ13464, } \\
\text { ULLZ13467 }\end{array}$ & BOLD:ACG7379 & 4 & - & - \\
\hline Mithracidae & $\begin{array}{l}\text { Mithraculus } \\
\text { coryphe }\end{array}$ & $\begin{array}{l}\text { ULLZ13318, } \\
\text { ULLZ13460, } \\
\text { ULLZ13461, } \\
\text { ULLZ13621 }\end{array}$ & BOLD:ACT9266* & 4 & - & - \\
\hline Mithracidae & $\begin{array}{l}\text { Mithraculus } \\
\text { forceps }\end{array}$ & $\begin{array}{l}\text { ULLZ13361, } \\
\text { ULLZ13363, } \\
\text { ULLZ13571, } \\
\text { ULLZ13675, } \\
\text { ULLZ13568, } \\
\text { ULLZ13569 }\end{array}$ & BOLD:AAC9888 & 6 & - & - \\
\hline Mithracidae & Mithrax hispidus & $\begin{array}{l}\text { ULLZ13391, } \\
\text { ULLZ13453, } \\
\text { ULLZ13682 }\end{array}$ & BOLD:ACU0360* & 3 & - & - \\
\hline Mithracidae & $\begin{array}{l}\text { Mithrax } \\
\text { pleuracanthus }\end{array}$ & $\begin{array}{l}\text { ULLZ13348, } \\
\text { ULLZ13362, } \\
\text { ULLZ13435, } \\
\text { ULLZ13705 }\end{array}$ & BOLD:ACB5456 & 4 & - & NEW \\
\hline
\end{tabular}




\begin{tabular}{|c|c|c|c|c|c|c|}
\hline Family & Morphospecies & Museum ID & BINs & \#Inds & $\begin{array}{l}\text { COI new } \\
\text { to } \\
\text { GenBank } \\
\text { (<95\% } \\
\text { identity) }\end{array}$ & $\begin{array}{l}16 S \text { new } \\
\text { to } \\
\text { GenBank } \\
\text { (<97\% } \\
\text { identity) }\end{array}$ \\
\hline Mithracidae & $\begin{array}{l}\text { Omalacantha } \\
\text { bicornuta }\end{array}$ & $\begin{array}{l}\text { ULLZ13377, } \\
\text { ULLZ13378, } \\
\text { ULLZ13562, } \\
\text { ULLZ13563, } \\
\text { ULLZ13564, } \\
\text { ULLZ13567, } \\
\text { ULLZ13662, } \\
\text { ULLZ13376, } \\
\text { ULLZ13565, } \\
\text { ULLZ13566 }\end{array}$ & BOLD:AAX4083 & 10 & - & - \\
\hline Ocypodidae & Minuca burgersi & ULLZ13367 & BOLD:ACG7755 & 1 & - & - \\
\hline Ocypodidae & Minuca rapax & $\begin{array}{l}\text { ULLZ13366, } \\
\text { ULLZ13368, } \\
\text { ULLZ13441, } \\
\text { ULLZ13442 }\end{array}$ & BOLD:ACT8667 & 4 & - & - \\
\hline Ocypodidae & Ocypode quadrata & ULLZ13411 & BOLD:ACU0659 & 1 & - & - \\
\hline Panopeidae & $\begin{array}{l}\text { Acantholobulus } \\
\text { bermudensis }\end{array}$ & $\begin{array}{l}\text { ULLZ13329a, } \\
\text { ULLZ13329b }\end{array}$ & BOLD:ACG8166 & 2 & - & - \\
\hline Panopeidae & $\begin{array}{l}\text { Eurypanopeus } \\
\text { abbreviatus }\end{array}$ & ULLZ13590 & BOLD:ACU0495 & 1 & - & - \\
\hline Panopeidae & Eurytium limosum & $\begin{array}{l}\text { ULLZ13382, } \\
\text { ULLZ13383, } \\
\text { ULLZ13473 }\end{array}$ & BOLD:ACT8759 & 3 & - & - \\
\hline Panopeidae & Panopeus lacustris & ULLZ13686 & BOLD:ACU0442 & 1 & - & - \\
\hline Panopeidae & $\begin{array}{l}\text { Panopeus } \\
\text { occidentalis }\end{array}$ & $\begin{array}{l}\text { ULLZ13344, } \\
\text { ULLZ13694 }\end{array}$ & BOLD:AAX2632 & 2 & - & - \\
\hline Percnidae & Percnon gibbesi & $\begin{array}{l}\text { ULLZ13443, } \\
\text { ULLZ13479 }\end{array}$ & BOLD:AAC3992 & 2 & - & - \\
\hline Pilumnidae & $\begin{array}{l}\text { Pilumnus } \\
\text { caribaeus }\end{array}$ & $\begin{array}{l}\text { ULLZ13393, } \\
\text { ULLZ13394 }\end{array}$ & BOLD:ACG8072 & 2 & - & - \\
\hline
\end{tabular}




\begin{tabular}{|c|c|c|c|c|c|c|}
\hline Family & Morphospecies & Museum ID & BINs & \#Inds & $\begin{array}{l}\text { COI new } \\
\text { to } \\
\text { GenBank } \\
\text { (<95\% } \\
\text { identity) }\end{array}$ & $\begin{array}{l}16 S \text { new } \\
\text { to } \\
\text { GenBank } \\
\text { (<97\% } \\
\text { identity) }\end{array}$ \\
\hline Pilumnidae & $\begin{array}{l}\text { Pilumnus } \\
\text { dasypodus, } P \text {. } \\
\text { caribaeus }\end{array}$ & $\begin{array}{l}\text { ULLZ13440, } \\
\text { ULLZ13616, } \\
\text { ULLZ13439, } \\
\text { ULLZ13614, } \\
\text { ULLZ13615 }\end{array}$ & BOLD:AAI2968 & 5 & - & - \\
\hline Pilumnidae & $\begin{array}{l}\text { Pilumnus } \\
\text { gemmatus }\end{array}$ & $\begin{array}{l}\text { ULLZ13462, } \\
\text { ULLZ13680, } \\
\text { ULLZ13681 }\end{array}$ & BOLD:AAY4016 & 3 & - & - \\
\hline Pilumnidae & $\begin{array}{l}\text { Pilumnus } \\
\text { holosericus }\end{array}$ & $\begin{array}{l}\text { ULLZ13577, } \\
\text { ULLZ13578 }\end{array}$ & BOLD:ACU1343 & 2 & - & - \\
\hline Pilumnidae & $\begin{array}{l}\text { Pilumnus } \\
\text { nudimanus }\end{array}$ & ULLZ13466 & BOLD:ACT9365 & 1 & - & - \\
\hline Pilumnidae & $\begin{array}{l}\text { Pilumnus } \\
\text { pannosus, } P \text {. } \\
\text { reticulatus }\end{array}$ & $\begin{array}{l}\text { ULLZ13581, } \\
\text { ULLZ13589 }\end{array}$ & & 2 & - & - \\
\hline Pinnotheridae & Austinixa aidae & ULLZ13459 & BOLD:ACU0215* & 1 & NEW & - \\
\hline Pinnotheridae & Austinixa sp. & $\begin{array}{l}\text { ULLZ13644, } \\
\text { ULLZ13642, } \\
\text { ULLZ13643, } \\
\text { ULLZ13332 }\end{array}$ & $\begin{array}{l}\text { BOLD:ACU0214*, } \\
\text { BOLD:ACU0213* }\end{array}$ & 4 & NEW & - \\
\hline Pinnotheridae & $\begin{array}{l}\text { Tunicotheres } \\
\text { moseri^ }\end{array}$ & ULLZ13678 & BOLD:ACT9535* & 1 & NEW & - \\
\hline Plagusiidae & $\begin{array}{l}\text { Plagusia depressa } \\
\wedge\end{array}$ & ULLZ13403 & BOLD:ACT9499* & 1 & NEW & - \\
\hline Portunidae & Achelous sebae ${ }^{\wedge}$ & ULLZ13477 & BOLD:ACG7575 & 1 & NEW & - \\
\hline Portunidae & Callinectes danae & $\begin{array}{l}\text { ULLZ13321, } \\
\text { ULLZ13373, } \\
\text { ULLZ13409, } \\
\text { ULLZ13410, } \\
\text { ULLZ13700 }\end{array}$ & BOLD:ACD2797 & 5 & - & - \\
\hline
\end{tabular}




\begin{tabular}{|c|c|c|c|c|c|c|}
\hline Family & Morphospecies & Museum ID & BINs & \#Inds & $\begin{array}{l}\text { COI new } \\
\text { to } \\
\text { GenBank } \\
\text { (<95\% } \\
\text { identity) }\end{array}$ & $\begin{array}{l}16 S \text { new } \\
\text { to } \\
\text { GenBank } \\
\text { (<97\% } \\
\text { identity) }\end{array}$ \\
\hline Portunidae & $\begin{array}{l}\text { Callinectes } \\
\text { larvatus }\end{array}$ & $\begin{array}{l}\text { ULLZ13372, } \\
\text { ULLZ13392, } \\
\text { ULLZ13405, } \\
\text { ULLZ13406, } \\
\text { ULLZ13407, } \\
\text { ULLZ13476 }\end{array}$ & BOLD:ACC4630 & 6 & - & - \\
\hline Portunidae & Charybdis hellerii & $\begin{array}{l}\text { ULLZ13355, } \\
\text { ULLZ13465, } \\
\text { ULLZ13584, } \\
\text { ULLZ13585 }\end{array}$ & BOLD:AAO9264 & 4 & - & - \\
\hline Pseudothelphusidae & Ptychophallus sp. & $\begin{array}{l}\text { ULLZ13471, } \\
\text { ULLZ13684 }\end{array}$ & BOLD:ACU0372 & 2 & - & - \\
\hline Sesarmidae & Aratus pisonii & $\begin{array}{l}\text { ULLZ13365, } \\
\text { ULLZ13364 }\end{array}$ & BOLD:ACG8032 & 2 & - & - \\
\hline Sesarmidae & Armases ricordi & $\begin{array}{l}\text { ULLZ13452, } \\
\text { ULLZ13579, } \\
\text { ULLZ13580 }\end{array}$ & BOLD:ACT9799* & 3 & NEW & - \\
\hline Sesarmidae & $\begin{array}{l}\text { Sesarma } \\
\text { curacaoense }\end{array}$ & $\begin{array}{l}\text { ULLZ13294, } \\
\text { ULLZ13388, } \\
\text { ULLZ13389, } \\
\text { ULLZ13390 }\end{array}$ & BOLD:ACT9653 & 4 & - & - \\
\hline Varunidae & $\begin{array}{l}\text { Cyclograpsus } \\
\text { integer }\end{array}$ & $\begin{array}{l}\text { ULLZ13431, } \\
\text { ULLZ13691 }\end{array}$ & BOLD:ACT8869 & 2 & - & - \\
\hline Xanthidae & $\begin{array}{l}\text { Cataleptodius } \\
\text { floridanus }\end{array}$ & $\begin{array}{l}\text { ULLZ13349, } \\
\text { ULLZ13417, } \\
\text { ULLZ13418, } \\
\text { ULLZ13419 }\end{array}$ & BOLD:AAI1248 & 4 & - & - \\
\hline Xanthidae & Paraliomera dispar & ULLZ13413 & BOLD:ACH4832 & 1 & - & - \\
\hline \multicolumn{7}{|c|}{ Shrimps: Caridea, Dendrobranchiata and Stenopodidea } \\
\hline Alpheidae & Alpheus sp. & & BOLD:AAH8594 & 1 & - & - \\
\hline Alpheidae & Alpheus armillatus & $\begin{array}{l}\text { OUMNH:ZC: } \\
2008-14-117\end{array}$ & BOLD:ADP1810* & 1 & NEW & - \\
\hline
\end{tabular}




\begin{tabular}{|c|c|c|c|c|c|c|}
\hline Family & Morphospecies & Museum ID & BINs & \#lnds & $\begin{array}{l}\text { COI new } \\
\text { to } \\
\text { GenBank } \\
\text { (<95\% } \\
\text { identity) }\end{array}$ & $\begin{array}{l}16 S \text { new } \\
\text { to } \\
\text { GenBank } \\
\text { (<97\% } \\
\text { identity) }\end{array}$ \\
\hline Alpheidae & Alpheus angulosus & $\begin{array}{l}\text { OUMNH:ZC: } \\
\text { 2008-14-082, } \\
\text { OUMNH:ZC: } \\
\text { 2008-14-095, } \\
\text { OUMNH:ZC: } \\
\text { 2008-14-100, } \\
\text { ULLZ13652 }\end{array}$ & BOLD:AAC6145* & 11 & - & - \\
\hline Alpheidae & $\begin{array}{l}\text { Alpheus } \\
\text { bahamensis }\end{array}$ & $\begin{array}{l}\text { OUMNH:ZC: } \\
\text { 2008-14-079, } \\
\text { OUMNH:ZC: } \\
\text { 2008-14-080, } \\
\text { ULLZ13645, } \\
\text { ULLZ13646, } \\
\text { ULLZ13647 }\end{array}$ & BOLD:AAC6728* & 9 & NEW & NEW \\
\hline Alpheidae & $\begin{array}{l}\text { Alpheus } \\
\text { cristulifrons }\end{array}$ & $\begin{array}{l}\text { OUMNH:ZC: } \\
\text { 2008-14-089, } \\
\text { OUMNH:ZC: } \\
\text { 2009-14-084 }\end{array}$ & BOLD:ADP1409* & 3 & - & NEW \\
\hline Alpheidae & $\begin{array}{l}\text { Alpheus } \\
\text { estuariensis }\end{array}$ & $\begin{array}{l}\text { OUMNH:ZC: } \\
\text { 2008-14-083 }\end{array}$ & BOLD:ADC6108 & 1 & - & - \\
\hline Alpheidae & Alpheus floridanus & ULLZ13478 & BOLD:ACU1957* & 1 & NEW & - \\
\hline Alpheidae & Alpheus nuttingi & $\begin{array}{l}\text { ULLZ13314A, } \\
\text { ULLZ13314B, } \\
\text { ULLZ13622, } \\
\text { ULLZ13625, } \\
\text { ULLZ13649, } \\
\text { ULLZ13650, } \\
\text { ULLZ13624 }\end{array}$ & BOLD:ACU0031* & 7 & - & - \\
\hline Alpheidae & $\begin{array}{l}\text { Alpheus packardii } \\
\text { complex }^{\wedge}\end{array}$ & $\begin{array}{l}\text { OUMNH:ZC: } \\
\text { 2008-14-081, } \\
\text { OUMNH:ZC: } \\
\text { 2008-14-108, } \\
\text { OUMNH:ZC: } \\
\text { 2008-14-111 }\end{array}$ & BOLD:ACQ5750 & 6 & - & NEW \\
\hline Alpheidae & $\begin{array}{l}\text { Alpheus packardii } \\
\text { complex }^{\wedge}\end{array}$ & $\begin{array}{l}\text { OUMNH:ZC: } \\
\text { 2008-14-081 }\end{array}$ & BOLD:AAC6138 & 1 & NEW & NEW \\
\hline
\end{tabular}




\begin{tabular}{|c|c|c|c|c|c|c|}
\hline Family & Morphospecies & Museum ID & BINs & \#Inds & $\begin{array}{l}\text { COI new } \\
\text { to } \\
\text { GenBank } \\
\text { (<95\% } \\
\text { identity) }\end{array}$ & $\begin{array}{l}16 S \text { new } \\
\text { to } \\
\text { GenBank } \\
\text { (<97\% } \\
\text { identity) }\end{array}$ \\
\hline Alpheidae & $\begin{array}{l}\text { Alpheus packardii } \\
\text { complex sp. } 1^{\wedge}\end{array}$ & $\begin{array}{l}\text { OUMNH:ZC: } \\
\text { 2008-14-084, } \\
\text { OUMNH:ZC: } \\
\text { 2008-14-085, } \\
\text { ULLZ13447, } \\
\text { ULLZ13626, } \\
\text { ULLZ13628, } \\
\text { ULLZ13630, } \\
\text { ULLZ13631, } \\
\text { OUMNH:ZC: } \\
\text { 2008-14-081 }\end{array}$ & BOLD:AAH7067* & 9 & NEW & NEW \\
\hline Alpheidae & $\begin{array}{l}\text { Alpheus packardii } \\
\text { complex sp. } 2^{\wedge}\end{array}$ & ULLZ13627 & BOLD:ACT9784* & 1 & NEW & NEW \\
\hline Alpheidae & $\begin{array}{l}\text { Alpheus } \\
\text { paracrinitus }\end{array}$ & $\begin{array}{l}\text { OUMNH:ZC: } \\
\text { 2008-14-118, } \\
\text { OUMNH:ZC: } \\
\text { 2018-14-105, } \\
\text { OUMNH:ZC: } \\
\text { 2018-14-107 }\end{array}$ & BOLD:ADP3337* & 4 & NEW & NEW \\
\hline Alpheidae & $\begin{array}{l}\text { Alpheus } \\
\text { paracrinitus }\end{array}$ & $\begin{array}{l}\text { OUMNH:ZC: } \\
\text { 2008-14-118 }\end{array}$ & BOLD:ADP0639* & 1 & NEW & NEW \\
\hline Alpheidae & $\begin{array}{l}\text { Alpheus } \\
\text { paraformosus }\end{array}$ & $\begin{array}{l}\text { OUMNH:ZC: } \\
\text { 2008-14-087 }\end{array}$ & BOLD:AAC6141 & 1 & - & - \\
\hline Alpheidae & Alpheus peasei & $\begin{array}{l}\text { OUMNH:ZC: } \\
\text { 2008-14-086, } \\
\text { OUMNH:ZC: } \\
\text { 2008-14-090, } \\
\text { OUMNH:ZC: } \\
\text { 2008-14-114: }\end{array}$ & BOLD:ADP2822* & 3 & - & NEW \\
\hline Alpheidae & Alpheus thomasi & $\begin{array}{l}\text { ULLZ13320, } \\
\text { ULLZ13629 }\end{array}$ & BOLD:ACT9880 & 2 & - & - \\
\hline Alpheidae & Alpheus viridari & $\begin{array}{l}\text { OUMNH:ZC: } \\
\text { 2008-14-094, } \\
\text { OUMNH:ZC: } \\
\text { 2008-14-110, } \\
\text { ULLZ13677 }\end{array}$ & BOLD:AAI2078 & 3 & - & - \\
\hline
\end{tabular}




\begin{tabular}{|c|c|c|c|c|c|c|}
\hline Family & Morphospecies & Museum ID & BINs & \#lnds & $\begin{array}{l}\text { COI new } \\
\text { to } \\
\text { GenBank } \\
\text { (<95\% } \\
\text { identity) }\end{array}$ & $\begin{array}{l}16 \mathrm{~S} \text { new } \\
\text { to } \\
\text { GenBank } \\
\text { (<97\% } \\
\text { identity) }\end{array}$ \\
\hline Alpheidae & $\begin{array}{l}\text { Automate aff. } \\
\text { dolichognatha }^{\wedge}\end{array}$ & $\begin{array}{l}\text { OUMNH:ZC: } \\
\text { 2008-14-109 }\end{array}$ & BOLD:ADO9733* & 1 & NEW & NEW \\
\hline Alpheidae & $\begin{array}{l}\text { Synalpheus } \\
\text { brevicarpus }\end{array}$ & $\begin{array}{l}\text { ULLZ13639, } \\
\text { ULLZ13640 }\end{array}$ & BOLD:ACC9307 & 2 & - & - \\
\hline Alpheidae & $\begin{array}{l}\text { Synalpheus } \\
\text { fritzmuelleri }\end{array}$ & $\begin{array}{l}\text { OUMNH:ZC: } \\
\text { 2008-14-127 }\end{array}$ & $\begin{array}{l}\text { BOLD:ADP1424*, } \\
\text { BOLD:AAG9019 }\end{array}$ & 3 & NEW & NEW \\
\hline Alpheidae & $\begin{array}{l}\text { Synalpheus } \\
\text { hemphilli }\end{array}$ & ULLZ13641 & BOLD:ADP2538* & 3 & NEW & NEW \\
\hline Alpheidae & Synalpheus yano & $\begin{array}{l}\text { OUMNH:ZC: } \\
\text { 2008-14-133 }\end{array}$ & BOLD:AAC6139 & 24 & - & - \\
\hline Alpheidae & $\begin{array}{l}\text { Synalpheus nr. } \\
\text { yano }\end{array}$ & & BOLD:ADP3823* & 4 & NEW & NEW \\
\hline Alpheidae & $\begin{array}{l}\text { Synalpheus } n r . \\
\text { yano }\end{array}$ & $\begin{array}{l}\text { OUMNH:ZC: } \\
\text { 2008-14-133 }\end{array}$ & BOLD:ACC9017 & 2 & - & NEW \\
\hline Alpheidae & $\begin{array}{l}\text { Synalpheus nr. } \\
\text { yano }\end{array}$ & & BOLD:ACC9109 & 2 & - & - \\
\hline Alpheidae & $\begin{array}{l}\text { Synalpheus } n r . \\
\text { yano }\end{array}$ & $\begin{array}{l}\text { OUMNH:ZC: } \\
\text { 2008-14-103 }\end{array}$ & BOLD:AAC6142 & 1 & - & - \\
\hline Alpheidae & $\begin{array}{l}\text { Synalpheus nr. } \\
\text { yano }\end{array}$ & & BOLD:ADP1425 & 1 & - & - \\
\hline Alpheidae & $\begin{array}{l}\text { Synalpheus nr. } \\
\text { yano }\end{array}$ & $\begin{array}{l}\text { OUMNH:ZC: } \\
\text { 2008-14-133 }\end{array}$ & BOLD:ADP4347* & 6 & - & - \\
\hline Alpheidae & $\begin{array}{l}\text { Synalpheus nr. } \\
\text { yano }\end{array}$ & $\begin{array}{l}\text { OUMNH:ZC: } \\
\text { 2008-14-133 }\end{array}$ & BOLD:ADP4348* & 2 & - & - \\
\hline Alpheidae & $\begin{array}{l}\text { Synalpheus } \\
\text { dardeaui }\end{array}$ & $\begin{array}{l}\text { OUMNH:ZC: } \\
\text { 2008-14-133, } \\
\text { ULLZ13488 }\end{array}$ & BOLD:AAE5682 & 10 & - & - \\
\hline Alpheidae & $\begin{array}{l}\text { Synalpheus ul, S. } \\
\text { longicarpus }\end{array}$ & $\begin{array}{l}\text { OUMNH:ZC: } \\
\text { 2008-14-127, } \\
\text { ULLZ13710 }\end{array}$ & BOLD:AAG9018 & 4 & - & - \\
\hline Alpheidae & $\begin{array}{l}\text { Synalpheus } \\
\text { apioceros }\end{array}$ & $\begin{array}{l}\text { OUMNH:ZC: } \\
\text { 2008-14-091 }\end{array}$ & $\begin{array}{l}\text { BOLD:ADO8257*, } \\
\text { BOLD:AAD6588 }\end{array}$ & 5 & - & NEW \\
\hline Alpheidae & Synalpheus guerini & & BOLD:AAG9015 & 3 & - & - \\
\hline
\end{tabular}




\begin{tabular}{|c|c|c|c|c|c|c|}
\hline Family & Morphospecies & Museum ID & BINs & \#lnds & $\begin{array}{l}\text { COI new } \\
\text { to } \\
\text { GenBank } \\
\text { (<95\% } \\
\text { identity) }\end{array}$ & $\begin{array}{l}16 S \text { new } \\
\text { to } \\
\text { GenBank } \\
\text { (<97\% } \\
\text { identity) }\end{array}$ \\
\hline Alpheidae & $\begin{array}{l}\text { Synalpheus } \\
\text { scaphoceris }\end{array}$ & & BOLD:AAF9341 & 4 & - & - \\
\hline Atyidae & Potimirim glabra & $\begin{array}{l}\text { OUMNH:ZC: } \\
\text { 2008-14-014 }\end{array}$ & BOLD:ACI0486 & 1 & - & - \\
\hline Hippolytidae & $\begin{array}{l}\text { Hippolyte } \\
\text { obliquimanus }\end{array}$ & $\begin{array}{l}\text { OUMNH:ZC: } \\
\text { 2008-14-043, } \\
\text { OUMNH:ZC: } \\
\text { 2008-14-045, } \\
\text { ULLZ13696, } \\
\text { ULLZ13697 }\end{array}$ & BOLD:AAE4017 & 7 & - & - \\
\hline Hippolytidae & $\begin{array}{l}\text { Tozeuma } \\
\text { carolinense }\end{array}$ & $\begin{array}{l}\text { OUMNH:ZC: } \\
\text { 2008-14-051, } \\
\text { ULLZ13380, } \\
\text { ULLZ13381, } \\
\text { ULLZ13379 }\end{array}$ & BOLD:ACU0079* & 8 & NEW & - \\
\hline Hippolytidae & $\begin{array}{l}\text { Tozeuma } \\
\text { carolinense }\end{array}$ & $\begin{array}{l}\text { OUMNH:ZC: } \\
\text { 2008-14-051 }\end{array}$ & BOLD:ADP3533* & 1 & NEW & NEW \\
\hline Palaemonidae & $\begin{array}{l}\text { Brachycarpus } \\
\text { biunguiculatus }\end{array}$ & $\begin{array}{l}\text { OUMNH:ZC: } \\
\text { 2008-14-038, } \\
\text { OUMNH:ZC: } \\
\text { 2008-14-068 }\end{array}$ & BOLD:AAE0296 & 2 & NEW & - \\
\hline Palaemonidae & $\begin{array}{l}\text { Cuapetes } \\
\text { americanus }\end{array}$ & $\begin{array}{l}\text { ULLZ13670, } \\
\text { OUMNH:ZC: } \\
\text { 2008-14-032, } \\
\text { ULLZ13668, } \\
\text { ULLZ13671, } \\
\text { ULLZ13712, } \\
\text { ULLZ13715, } \\
\text { ULLZ13669, } \\
\text { ULLZ13713 }\end{array}$ & $\begin{array}{l}\text { BOLD:ACG8330, } \\
\text { BOLD:AAI2206 }\end{array}$ & 10 & - & - \\
\hline Palaemonidae & Leander paulensis & \multicolumn{2}{|l|}{ ULLZ13685 } & 1 & - & - \\
\hline
\end{tabular}




\begin{tabular}{|c|c|c|c|c|c|c|}
\hline Family & Morphospecies & Museum ID & BINs & \#Inds & $\begin{array}{l}\text { COI new } \\
\text { to } \\
\text { GenBank } \\
\text { (<95\% } \\
\text { identity) }\end{array}$ & $\begin{array}{l}16 S \text { new } \\
\text { to } \\
\text { GenBank } \\
\text { (<97\% } \\
\text { identity) }\end{array}$ \\
\hline Palaemonidae & $\begin{array}{l}\text { Leander } \\
\text { tenuicornis }\end{array}$ & $\begin{array}{l}\text { OUMNH:ZC: } \\
\text { 2008-14-034, } \\
\text { OUMNH:ZC: } \\
\text { 2008-14-036, } \\
\text { OUMNH:ZC: } \\
\text { 2008-14-067, } \\
\text { ULLZ13351, } \\
\text { ULLZ13485a, } \\
\text { ULLZ13485b, } \\
\text { ULLZ13486a, } \\
\text { ULLZ13486b }\end{array}$ & BOLD:AAC8465 & 10 & NEW & - \\
\hline Palaemonidae & $\begin{array}{l}\text { Palaemon } \\
\text { northropi }\end{array}$ & $\begin{array}{l}\text { OUMNH:ZC: } \\
\text { 2008-14-031, } \\
\text { ULLZ13356 }\end{array}$ & BOLD:AAG9010 & 2 & - & - \\
\hline Palaemonidae & $\begin{array}{l}\text { Periclimenaeus } \\
\text { schmitti^ } ~\end{array}$ & $\begin{array}{l}\text { OUMNH:ZC: } \\
\text { 2008-14-064, } \\
\text { OUMNH:ZC: } \\
\text { 2008-14-072, } \\
\text { OUMNH:ZC: } \\
\text { 2008-14-073, } \\
\text { OUMNH:ZC: } \\
\text { 2008-14-074, } \\
\text { OUMNH:ZC: } \\
\text { 2008-14-075, } \\
\text { OUMNH:ZC: } \\
\text { 2008-14-076, } \\
\text { OUMNH:ZC: } \\
2008-14-139\end{array}$ & BOLD:ADP1635* & 8 & NEW & NEW \\
\hline Palaemonidae & $\begin{array}{l}\text { Periclimenes } \\
\text { rathbunae }\end{array}$ & ULLZ13699 & BOLD:AAC6144 & 1 & - & - \\
\hline Palaemonidae & $\begin{array}{l}\text { Periclimenes } \\
\text { yucatanicus }\end{array}$ & $\begin{array}{l}\text { ULLZ13472, } \\
\text { ULLZ13345, } \\
\text { ULLZ13704, } \\
\text { ULLZ13346, } \\
\text { ULLZ13695 }\end{array}$ & $\begin{array}{l}\text { BOLD:ADC8100*, } \\
\text { BOLD:ADC8099*, } \\
\text { BOLD:ACU2547*, } \\
\text { BOLD:AAH8593* }\end{array}$ & 5 & - & - \\
\hline Palaemonidae & Typton carneus ${ }^{\wedge}$ & ULLZ13711 & BOLD:ACU1120* & 1 & NEW & NEW \\
\hline
\end{tabular}




\begin{tabular}{|c|c|c|c|c|c|c|}
\hline Family & Morphospecies & Museum ID & BINs & \#lnds & $\begin{array}{l}\text { COI new } \\
\text { to } \\
\text { GenBank } \\
\text { (<95\% } \\
\text { identity) }\end{array}$ & $\begin{array}{l}16 S \text { new } \\
\text { to } \\
\text { GenBank } \\
\text { (<97\% } \\
\text { identity) }\end{array}$ \\
\hline Palaemonidae & $\begin{array}{l}\text { Typton cf. } \\
\text { distinctus } \sim\end{array}$ & $\begin{array}{l}\text { ULLZ13448, } \\
\text { ULLZ13698 }\end{array}$ & BOLD:ACT9785* & 2 & NEW & NEW \\
\hline Penaeidae & $\begin{array}{l}\text { Metapenaeopsis } \\
\text { gerardoi }\end{array}$ & ULLZ13357 & BOLD:ACT9874 & 1 & NEW & NEW \\
\hline Processidae & $\begin{array}{l}\text { Processa } \\
\text { bermudensis^ }\end{array}$ & $\begin{array}{l}\text { OUMNH:ZC: } \\
\text { 2008-14-059, } \\
\text { ULLZ13333 }\end{array}$ & BOLD:AAJ2144* & 2 & NEW & - \\
\hline Processidae & $\begin{array}{l}\text { Processa fimbriata } \\
\wedge\end{array}$ & $\begin{array}{l}\text { OUMNH:ZC: } \\
\text { 2008-14-056 }\end{array}$ & BOLD:AAF3128 & 2 & NEW & NEW \\
\hline Sicyoniidae & Sicyonia sp. & & BOLD:ADO8841* & 2 & NEW & NEW \\
\hline Sicyoniidae & Sicyonia sp. & & BOLD:ADO8840* & 1 & NEW & - \\
\hline Sicyoniidae & Sicyonia laevigata & ULLZ13702 & BOLD:ACT9954 & 1 & - & - \\
\hline Sicyoniidae & Sicyonia laevigata & $\begin{array}{l}\text { ULLZ13484, } \\
\text { ULLZ13703 }\end{array}$ & BOLD:AAF9340 & 4 & - & - \\
\hline Spongicolidae & $\begin{array}{l}\text { Microprosthema } \\
\text { semilaeve }^{\wedge}\end{array}$ & $\begin{array}{l}\text { OUMNH:ZC: } \\
\text { 2008-14-052, } \\
\text { OUMNH:ZC: } \\
\text { 2008-14-053, } \\
\text { ULLZ13648, } \\
\text { ULLZ13716 }\end{array}$ & BOLD:AAD8095* & 4 & NEW & - \\
\hline Stenopodidae & Stenopus hispidus & $\begin{array}{l}\text { ULLZ13666, } \\
\text { OUMNH:ZC: } \\
\text { 2008-14-076, } \\
\text { ULLZ13676 }\end{array}$ & BOLD:AAC8463 & 3 & - & - \\
\hline Stenopodidae & $\begin{array}{l}\text { Stenopus } \\
\text { scutellatus }\end{array}$ & $\begin{array}{l}\text { OUMNH:ZC: } \\
2008-14-047\end{array}$ & BOLD:ADD4717 & 1 & - & - \\
\hline \multicolumn{7}{|c|}{ Mudshrimp: Axiidea } \\
\hline Callianassidae & $\begin{array}{l}\text { Neocallichirus } \\
\text { grandimana }^{\wedge}\end{array}$ & $\begin{array}{l}\text { NHMW 25285, } \\
\text { NHMW } 25286\end{array}$ & BOLD:AAG5150* & 2 & NEW & - \\
\hline Callianassidae & $\begin{array}{l}\text { Neocallichirus } \\
\text { guassutinga }\end{array}$ & $\begin{array}{l}\text { NHMW 25287, } \\
\text { ULLZ13322, } \\
\text { ULLZ13323, } \\
\text { ULLZ13324 }\end{array}$ & BOLD:AAN0153 & 4 & - & - \\
\hline
\end{tabular}




\begin{tabular}{|c|c|c|c|c|c|c|}
\hline Family & Morphospecies & Museum ID & BINs & \#Inds & $\begin{array}{l}\text { COI new } \\
\text { to } \\
\text { GenBank } \\
\text { (<95\% } \\
\text { identity) }\end{array}$ & $\begin{array}{l}16 S \text { new } \\
\text { to } \\
\text { GenBank } \\
\text { (<97\% } \\
\text { identity) }\end{array}$ \\
\hline Callianassidae & $\begin{array}{l}\text { Neocallichirus } \\
\text { maryae }^{\wedge}\end{array}$ & ULLZ13474 & BOLD:ACT9837* & 1 & NEW & - \\
\hline Callianassidae & $\begin{array}{l}\text { Pseudobiffarius } \\
\text { caesari^ }^{\circ}\end{array}$ & ULLZ13480 & BOLD:ACU0753* & 1 & NEW & NEW \\
\hline \multicolumn{7}{|c|}{ Mudshrimp: Gebiidea } \\
\hline Upogebiidae & $\begin{array}{l}\text { Upogebia } \\
\text { corallifora }^{\wedge}\end{array}$ & ULLZ13683 & BOLD:ACT9886* & 1 & NEW & NEW \\
\hline \multicolumn{7}{|c|}{ Lobsters: Achelata } \\
\hline Palinuridae & Panulirus argus & $\begin{array}{l}\text { ULLZ13319, } \\
\text { ULLZ13458 }\end{array}$ & $\begin{array}{l}\text { BOLD:ACD2165, } \\
\text { BOLD:AAL9182 }\end{array}$ & 2 & - & - \\
\hline
\end{tabular}

\section{DNA Sequencing}

Specimens from the shrimp course were extracted in Panama using a Biosprint 96 and a DNA Blood Kit (Qiagen) and the DNA extracts were shipped to the Smithsonian's Laboratories of Analytical Biology (LAB) for PCR and sequencing. For the decapod course, small pieces of tissue were preserved in $150 \mu \mathrm{l}$ of M2 extraction buffer (AutoGen), stored frozen and shipped to LAB for extraction and sequencing. Samples were extracted using an AutoGenprep 965 extraction robot after overnight digestion in AutoGen buffer with proteinase-K. We sequenced two gene fragments. The DNA barcode fragment of the cytochrome c oxidase subunit I (COI) was amplified using primarily the primer pair jgLCO1490/jgHCO2198 (Geller et al. 2013), although the pair dgLCO1490/dgHCO2198 (Meyer et al. 2005) was also used. The $10 \mu \mathrm{l}$ PCR mix included $1 \mu$ l Biolase Taq (Promega), $0.1 \mu \mathrm{l} \mathrm{BSA}$ and $0.3 \mu \mathrm{l}$ of each $10 \mathrm{mM}$ primer. For amplification and sequencing of $16 \mathrm{~S}$, the primer pair $16 \mathrm{~S}$ AR/16S BR (Palumbi et al. 1991) was used. The mix for $16 \mathrm{~S}$ was the same as for $\mathrm{COI}$ with the addition of $0.5 \mu \mathrm{l} 50 \mathrm{mM} \mathrm{MgCl}$. The annealing temperature for nearly all reactions for all three gene regions was $50^{\circ} \mathrm{C}$, although occasionally it was raised to $52^{\circ} \mathrm{C}$ when it appeared that co-amplification was occurring. Sequencing followed the methods described in Collin et al. (2018), Collin et al. (2019), and Morín et al. (2019).

\section{Analysis}

Sequences were screened for quality and contigs of forward and reverse sequences were produced using Sequencher 5.4.6 (Gene Codes). Only sequences with a length of more than $90 \%$ of the expected length and with a Phred quality score of at least 30 for more than $85 \%$ of the bases were combined into contigs and used for analyses. To check for potential 
contamination, sequences were compared within the BOLD workbench (www.boldsystems. org; Ratnasingham and Hebert 2007) to all taxa sequenced in our project; likewise, sequences were compared to publicly available sequences using BLASTn searches in GenBank. The few sequences with $>95 \%$ identity to non-decapods were eliminated from subsequent analyses. $\mathrm{CO}$ sequences were also checked with the methods of Song et al. (2008) to determine whether they displayed detectable pseudogene traits (Buhay 2009).

As DNA barcoding is usually a distance-based approach, we constructed a neighbourjoining tree (BIONJ, Gascuel 1997) with Jukes-Cantor distances to preliminarily recognise distinct OTUs. Neighbour-joining trees with Kimura's-two-parameters distances were also constructed and produced the same results as the Jukes-Cantor distances. The tree nodes were further verified with non-parametric bootstrapping, using the Felsenstein's method (Felsenstein 1985, Efron et al. 1996). COI alignments were inferred with the BOLD aligner [amino acid-based Hidden Markov Model (Ratnasingham and Hebert 2007)], whereas $16 \mathrm{~S}$ alignments used the Kalign algorithm (Lassmann and Sonnhammer 2005) with the default settings of the BOLD workbench. Alignments were subsequently corrected manually. Operational Taxonomic Units (OTUs) were delimited with the Automatic Barcode Gap Discovery method (Puillandre et al. 2011) using the following parameters: $P_{\min }=0.001 ; \mathrm{P}$ $\max =0.1$ for $\mathrm{COI}$ and 0.05 for $16 \mathrm{~S} ; \mathrm{X}=1.125$ for $\mathrm{COI}$ and 1.5 for $16 \mathrm{~S}$; Steps $=10$. $\mathrm{P}_{\min }$ and $P_{\max }$ were chosen with the help of a histogram of distances and $X$ was smaller in $\mathrm{CO}$ because the default 1.5 value did not provide enough sensitivity to partition the data (see Puillandre et al. 2011).

Whenever an OTU differed between COI and 16S, the OTU was accepted only if it diverged from every other sequence by at least 0.05 substitutions per site in $\mathrm{COI}$ or 0.03 in 16S. If the discrepancy remained unresolved, then we accepted the option producing fewer OTUs. The final consensus OTUs were compared to the system of Barcode Index Numbers (BINs) assigned in BOLD (Ratnasingham and Hebert 2013) and to our morphological identifications, in order to detect potentially cryptic species or previously unrecognised diversity.

\section{Data resources}

The DNA sequences associated with this paper are deposited in the Barcode of Life Database (dataset dx.doi.org/10.5883/DS-CRUSTACE) (Ratnasingham and Hebert 2007, Ratnasingham and Hebert 2013) and GenBank (www.ncbi.nlm.nih.gov/genbank) (accession numbers MN183805-MN184218 for COI and MK971234-MK971659 for 16S).

\section{Results and Discussion}

A total of 447 individuals, morphologically identified to 129 species, were successfully sequenced for at least one marker, including 47 species of shrimps, 57 brachyuran crabs, one achelate lobster, four axiid mudshrimp, one gebiid mudshrimp and 19 anomuran crabs (Table 1, Figs 1, 2). Shrimps included the infra-orders Caridea, Dendrobranchiata and 
Stenopodidea. Amongst successfully sequenced individuals, 99 were identified to genus, but could not be confidently assigned to a species based on morphology. The Automatic Barcode Gap Discovery method delimited 141 OTUs with COI and 140 OTUs with 16S; likewise, our $\mathrm{CO}$ sequences were assigned to 146 Barcode Index Numbers (BINs) in BOLD. The larger number of OTUs and BINs suggest there are $\sim 10$ potentially cryptic species or species with unusually high levels of genetic diversity in this dataset.

\section{Common order: Decapoda}

Alpheidae: 142

Palaemonidae: 42

Mithracidae: 32

Porcellanidae: 29

Diogenidae: 18

Epialtidae: 16

Hippolytidae: 16

\section{2 others: 152}

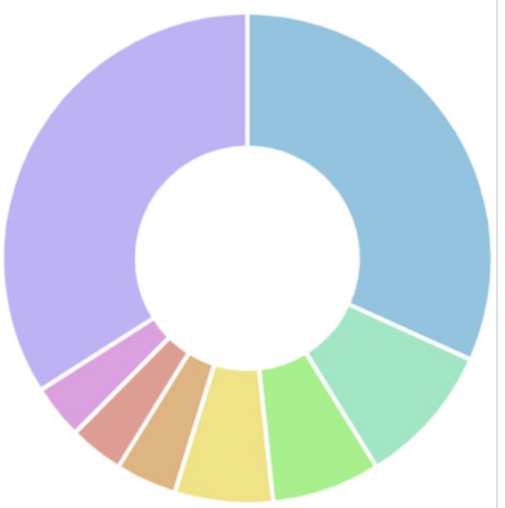

Figure 1. doi

Pie chart indicating the number of individuals present on this study for each decapod family.

Eighty seven of our consensus OTUs matched COI sequences already in GenBank with an identity of $>95 \%$ (see Table 1). Of these, our identification and the name on the GenBank sequence were concordant for 77 of the OTUs, including seven cases in which our identification provided better taxonomic resolution than the GenBank sequence. In many cases, these represent samples of the same taxa from other Caribbean regions confirming the conspecific status of animals from different parts of the same biogeographic region. In ten cases where our identification was not concordant with the name of a COI GenBank sequence $>95 \%$ identical, the discrepancy typically occurred at the species level while the higher taxonomic ranks remained concordant. Two OTUs did not have sequences in COI: one was a singleton identified as Leander paulensis and the other included two specimens identified as Pilumnus reticulatus and $P$. pannosus. The remaining 51 OTUs for which we have $\mathrm{COI}$ sequences were $<95 \%$ identical to another sequence in GenBank and therefore considered to be new additions.

The results for the $16 \mathrm{~S}$ analysis were relatively similar: 99 consensus OTUs were $>97 \%$ identical to $16 \mathrm{~S}$ sequences available in GenBank and for 89 of them, the morphological identification coincided with the name of the GenBank sequence, whereas the other ten OTUs showed discrepancies at the species level with the GenBank sequence, but remained concordant at higher taxonomic ranks. Four singleton OTUs, identified as Stenopus scutellatus, Inachoides sp., Austinixa aidae and Speleophorus nodosus did not have sequences in $16 \mathrm{~S}$. The remaining $16 \mathrm{~S}$ sequences (belonging to $37 \mathrm{OTUs}$ ) were > $97 \%$ similar to other sequences in GenBank; thus these were considered new additions. 


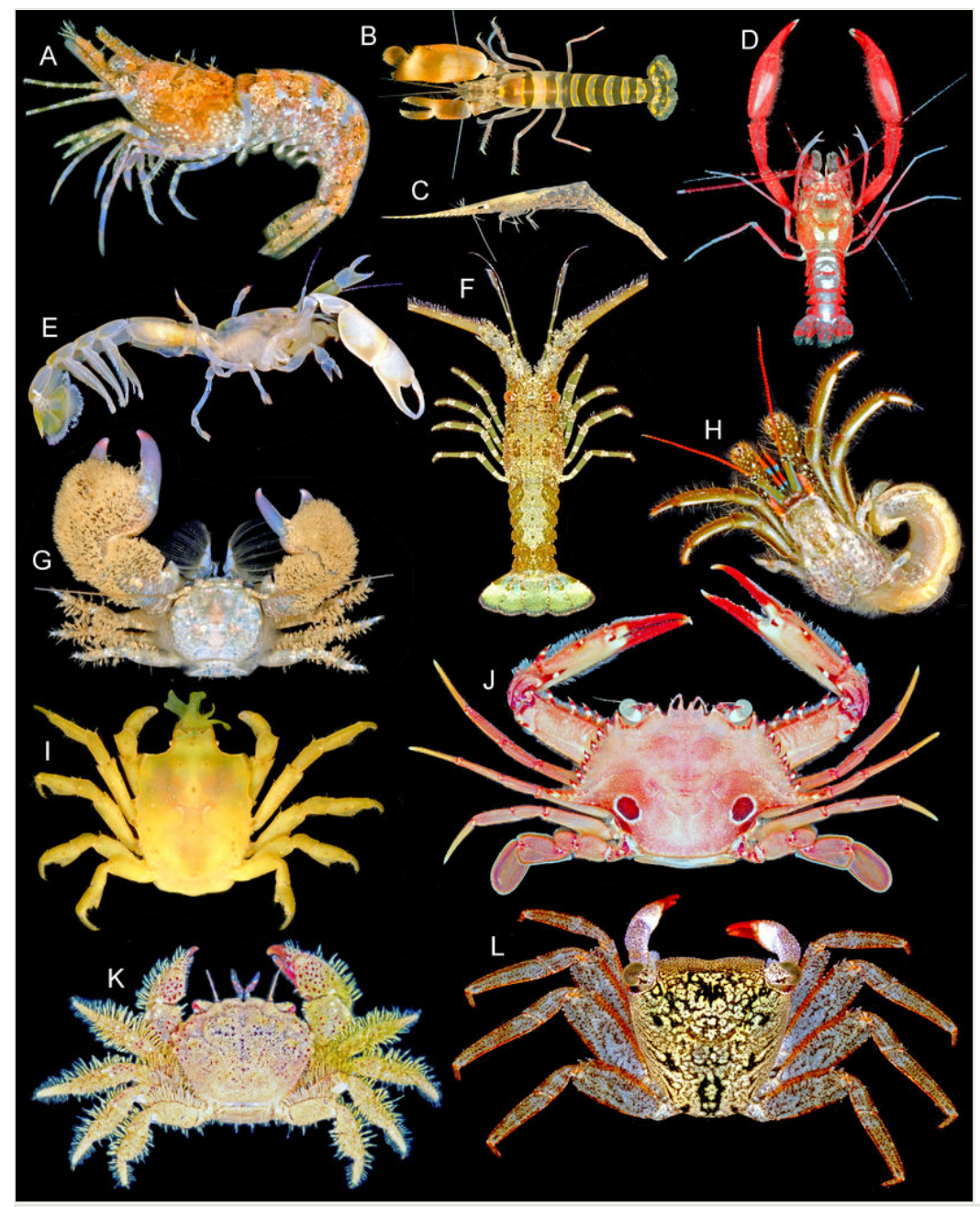

Figure 2. doi

Representative decapod crustacean specimens from Bocas del Toro, Panama. A. Sicyonia laevigata, ULLZ13484; B. Alpheus thomasi, ULLZ18292; C. Tozeuma carolinense, ULLZ18291; D. Microprosthema semilaeve, ULLZ10770; E. Pseudobiffarius caesari, ULLZ13480; F. Panulirus argus juvenile, ULLZ13319; G. Pachycheles tuerkayi, ULLZ6098; H. Clibanarius antillensis, ULLZ16971; I. Acanthonyx petiverii, ULLZ12015; J. Achelous sebae, ULLZ17128; K. Pilumnus holosericus, ULLZ17614; L. Aratus pisonii, ULLZ14799.

Our dataset contributed 56 new BINs to BOLD and provided 38 new species for at least one marker in GenBank (Table 1). One hundred and thirty seven of our 140 OTUs are associated with only one morphospecies name. This coincides with our visual observations of the $\mathrm{COI}$ and $16 \mathrm{~S}$ neighbour-joining trees, which showed that our morphospecies identifications are largely concordant with clusters of very similar sequences. These clusters differed from other such clusters by $\sim 0.10$ substitutions per site in $\mathrm{COI}$ and $\sim 0.05$ in 16S (Fig. 3). Such concordance between morphospecies and OTUs, and the magnitude 
of the observed interspecific divergence are similar to those reported by Costa et al. (2007) and Matzen da Silva et al. (2011). Nevertheless, there are several cases where animals could not be identified to species or in which a single species/species-complex name appeared in different OTUs. These included the following:

a
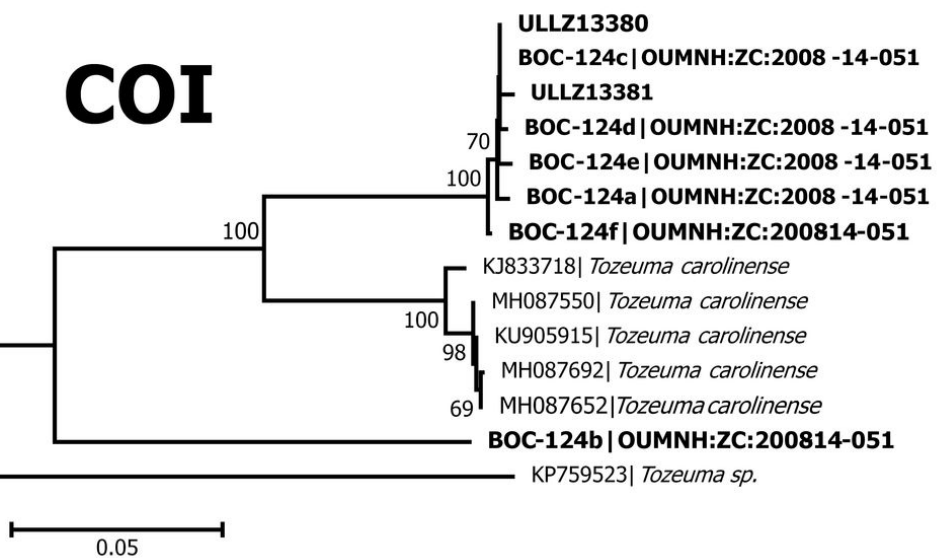

b

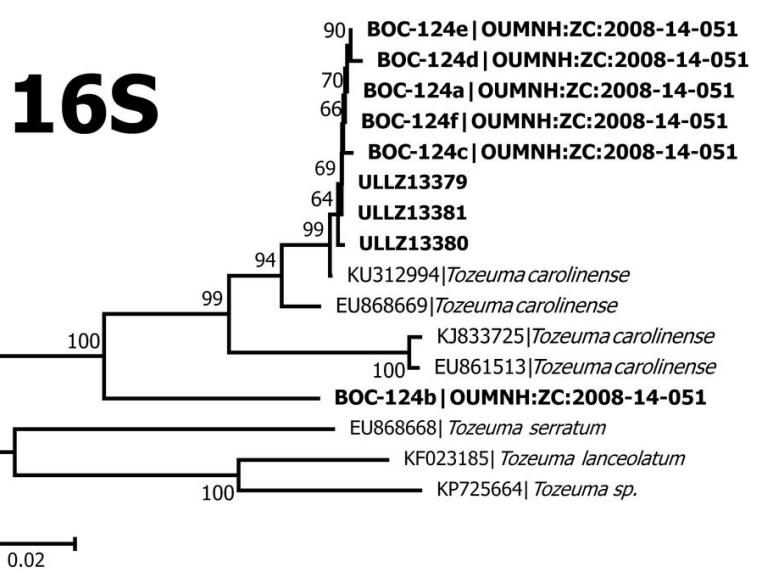

\section{Figure 3.}

Neighbour-Joining trees for cytochrome $c$ oxidase subunit I (COI) and 16S ribosomal RNA (16S) from specimens identified in this study and GenBank as Tozeuma carolinense. The accession number is provided for the GenBank sequences. Likewise, reference numbers are provided for specimens deposited in the University of Louisiana at Lafayette (ULLZ\#) and the Oxford University Museum of Natural History (OUMNH). The Jukes-Cantor distance between specimens is proportional to the length of the branches separating them, as indicated in the scale bars at the bottom-left.

a: Neighbour-Joining tree for cytochrome $c$ oxidase subunit I doi b: Neighbour-Joining tree for $16 S$ ribosomal RNA doi 
For Synalpheus spp., 42 individuals fell into eight OTUs. Seven OTUs matched GenBank sequences from in-depth studies of these taxa (Duffy et al. 2000, Morrison et al. 2004, Hultgren et al. 2014, Chak et al. 2017). These were S. hoetjesi, S. paraneptunus, S. yano, $S$. aff. longicarpus, S. elizabethae and $S$. cf. rathbunae. The final OTU did not match anything in GenBank (identity $<90 \%$ in both markers). Our failure to detect more than one additional species in this group suggests that it has been well-sampled in Bocas del Toro.

Specimens of Alpheus spp. were split into 18 OTUs. Thirteen OTUs were identified to species, including two OTUs assigned to the same species name (Alpheus paracrinitus); one of these OTUs had four individuals, whereas the other was a singleton. A. paracrinitus has long been considered an unresolved species complex, including at least four species (Knowlton et al. 1993, Anker 2001). The other five OTUs were identified to genus (Alpheus sp.) or as members of a species complex (e.g. A. packardii complex). Eleven Alpheus OTUs were new for BOLD, adding 11 new BINs to the database. Likewise, eleven Alpheus OTUs were $<95 \%$ identical to $\mathrm{COI}$ or $<97 \%$ to $16 \mathrm{~S}$ sequence in GenBank and, thus, constitute new additions to the database; moreover, our sequences added five new Alpheus species names for $16 \mathrm{~S}$ in GenBank. Only one OTU, identified as A. paraformosus, matched a different species name, $A$. formosus, in GenBank (sequence from Leray and Knowlton 2015). Most of the OTUs identified as members of the A. packardii complex are < $95 \%$ and $<97 \%$ identical to $\mathrm{COI}$ and $16 \mathrm{~S}$ sequence in GenBank, respectively. One other OTU, identified as Alpheus sp., matched GenBank sequences that were also identified only to genus. Clearly, we are far from having a complete barcode database for this speciose taxon.

The shrimps Tozeuma carolinense (Fig. 2C) fell into two OTUs, one with eight and the other with one specimen. Both of these OTUs were new for BOLD, adding two new BINs to the database. The $16 \mathrm{~S}$ sequences for one of these OTUs matched a $T$. carolinense sequence in GenBank with > 99\% identity. However, both of our T. carolinense OTUs were distinct from the available COI sequences in GenBank with > 95\% identity (Fig. 3, Table 1), suggesting that this morphologically distinctive species may include several cryptic species. A similar situation occurred for Sicyonia laevigata (Fig. 2A), Pagurus criniticornis and Alpheus paracrinitus; all these names being assigned to specimens that grouped in two OTUs: one with multiple species and the other being a singleton.

OTUs with multiple species names: One OTU included sequences from two specimens, morphologically identified as different species (Pilumnus dasypodus and $P$. caribaeus). This OTU matched $P$. dasypodus sequences in GenBank with $>99 \%$ identity for both markers. Another OTU identified as $P$. caribaeus in our dataset matched GenBank sequences of that species. One other OTU comprised specimens morphologically identified as 2 species ( $P$. pannosus and $P$. reticulatus).

\section{Pseudogenes in Decapod Barcoding}

We found no indels in our COI sequences and no stop codons in the corresponding amino acid sequence. Both the $\mathrm{COI}$ and $16 \mathrm{~S}$ sequences showed a range of GC content (GC\%) from $23.97-46.48 \%$ (Fig. 4), which is similar to the findings of other studies (e.g. Costa et 
al. 2007, Matzen da Silva et al. 2011). As mitochondrial genes are expected to show a different AT bias than pseudogenes (Bensasson 2001, Song et al. 2008, Matzen da Silva et al. 2011, Liu et al. 2016), those sequences with significantly deviant GC\% are potentially more likely to be pseudogenes; nevertheless, a careful examination of all our sequences failed to detect any strong evidence of pseudogenes. The overall concordance between our morphological identifications and the molecular identification of OTUs, based on $16 \mathrm{~S}$ and $\mathrm{COI}$, further supports the conclusion that pseudogenes were rare or absent in this dataset.

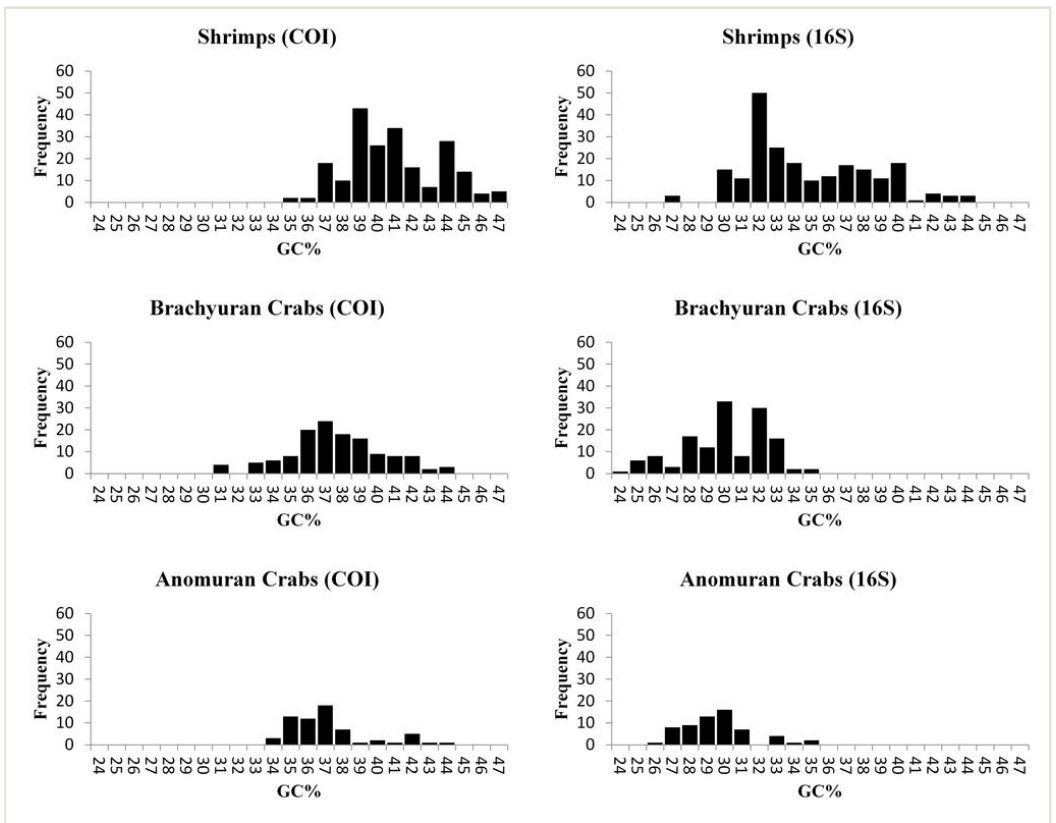

Figure 4. doi

Histogram of the GC content for COI and $16 \mathrm{~S}$ in three major groups of decapods evaluated in this study. Shrimps included the infra-orders Caridea, Dendrobranchiata and Stenopodidea.

Much has been made of the problem with pseudogenes in decapods (Schneider-Broussard and Neigel 1997, Williams and Knowlton 2001, Song et al. 2008, Schubart 2010, Matzen da Silva et al. 2011, Raupach and Radulovici 2015, but see Schizas 2012). They are undoubtedly more common in decapods than in some other groups of marine invertebrates, where they have seldom been reported. It is also clear that pseudogenes can cause significant problems in phylogenetic reconstructions (Schubart 2010). However, we argue that the problems they pose for DNA barcoding are limited and that the difficulty in determining if a sequence is a pseudogene or not without resorting to cloning, means that barcode datasets for decapods may never be entirely free of pseudogene sequences unless mitochondrial DNA is directly targeted during DNA extraction with a mitochondrial DNA isolation kit. 
Song et al. (2008) described a 3-step method for eliminating obvious pseudogenes in protein coding sequences: eliminate sequences with indels and stop codons, eliminate sequences with unusual or highly divergent amino acid sequences and eliminate sequences with unusual GC bias. The first two steps are only applicable to protein coding sequences like $\mathrm{COI}$ and cannot be applied to $16 \mathrm{~S}$, another commonly used barcoding gene for many groups [e.g. amphibians (Vences et al. 2005), hydrozoans (Zheng et al. 2014) and other marine invertebrates (Brasier et al. 2016)]. The third step is hindered by the difficulty in determining what GC\% value is sufficiently different to be considered suspect. The GC\% values of our sequences ranged from $24.0-46.5 \%$ and their histograms, in general, did not show any distinct gap or dip that could be considered as a clear threshold between coding sequences and pseudogenes. The small gaps and asymmetric distributions observed in some histograms were always associated with interspecific differences in GC\%; for example, the large GC\% of Dardanus fucosus, Calcinus tibicens and Paguristes tortugae explains the asymmetry and gaps in the anomuran histograms. A review of crustacean barcodes found that the GC\% varies significantly amongst families (Matzen da Silva et al. 2011), ranging from $\sim 30-50 \%$ with family-specific averages ranging from 33-42\% (Matzen da Silva et al. 2011). If AT/GC bias is similar across the mitochondrion, one might expect that the $\mathrm{GC} \%$ of $\mathrm{COI}$ and $16 \mathrm{~S}$ are correlated and deviations from the trend-line could be another way to identify potential pseudogenes. Our data show an overall positive correlation between the two, considerable scatter around the trend-line and a small cluster of carideans that fall somewhat below the other carideans, but still within the overall variation for the decapods (Fig. 5).

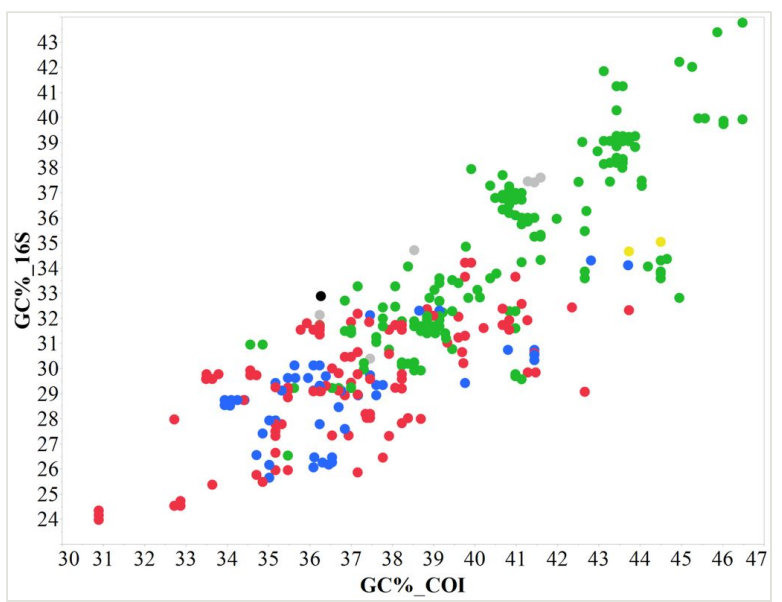

Figure 5. doi

Scatterplot of the GC content (GC\%) in COI versus $16 \mathrm{~S}$ for every individual of this study, successfully sequenced for both markers. In general, the GC\% of the two markers appears to be as correlated as expected for two mitochondrial genes and none of the specimens seems to deviate enough to be considered a suspect pseudogene. Major groups are indicated with colours: Brachyura: red, Anomura: blue, Shrimps (Caridea, Dendrobranchiata, and Stenopodidea): green, Axiidea: light-grey, Gebiidae: black, Achelata: yellow. 
Our limited ability to identify pseudogene sequences without cloning indicates that pseudogenes are likely to infiltrate metabarcoding datasets generated by high-throughput sequencing, as well as datasets generated by sanger sequencing. One concern about the inclusion of pseudogenes in these kinds of biodiversity studies is that they may overestimate the number of OTUs reported (Schneider-Broussard and Neigel 1997, Williams and Knowlton 2001, Song et al. 2008, Schubart 2010, Matzen da Silva et al. 2011, Raupach and Radulovici 2015). This could certainly happen, but a more common occurrence is that either co-amplification of the gene and its pseudogenes reduces the quality of the reads, resulting in an unusable sequence or a single sequence significantly out-amplifies the other, resulting in a single sequence from each species. If this is the pseudogene, the results could complicate phylogenetic analyses, but are unlikely to impact the results of DNA barcoding studies. One situation where pseudogenes certainly will not impact the efficacy of a DNA barcoding approach is in the identification of unknown samples through comparisons with sequences from carefully identified material. If a sequence is known to come from a specific species, whether or not it is a pseudogene, that sequence can be used to generate a positive identification of unknown material. Therefore, rather than discard potential pseudogene sequences, they should be included in barcode databases as a potentially informative resource (see also the arguments by Schizas 2012).

\section{Taxonomy Training and DNA Barcoding}

The present study, along with Cancian de Araujo et al. (2018), demonstrates that DNA barcoding of common species encountered during field training in tropical biodiversity can contribute useful data to the effort to barcode metazoans. Such data, despite collected from a single site, may be relevant throughout the Caribbean as connectivity is considered high within this sea for some decapods [e.g. Panulirus argus (Naro-Maciel et al. 2011, Kough et al. 2013)]. With only a moderate collecting effort (i.e. incidental collections over 4 weeks total), we obtained a barcode dataset of a similar number of decapod OTUs as the exhaustive decapod DNA barcode dataset for the North Sea (Raupach et al. 2015). Of these, $32 \%$ of the $\mathrm{COI}$ sequences and $24 \%$ of the $16 \mathrm{~S}$ sequences were new to GenBank and $39 \%$ of the BINs were new to BOLD. It has previously been noted that crustacean sequences are poorly represented in BOLD (Raupach and Radulovici 2015). The number of new OTUs is perhaps significantly lower than what would be expected in locations that have previously received less intensive systematic study than Bocas del Toro, which has been the focus of alpheid shrimp systematics for over 15 years (Williams and Knowlton 2001, Anker et al. 2007, Anker et al. 2008, Mathews and Anker 2009, Anker 2010, Anker et al. 2012). Nevertheless, a significant portion of the sequenced species generated new species records in GenBank and required little additional effort in the field over and above the collection and identification exercises already underway. Of course, as with any vouchered material, additional curatorial effort was necessary compared to typical field courses where the material is not usually vouchered. 


\section{Acknowledgements}

We thank the Autoridad de los Recursos Acuáticos de Panamá for issuing permits for this work and participants in the 2008 and 2011 workshops in Bocas del Toro. This research was supported by Paul Peck, a Grand Challenges Level 1 grant from the Smithsonian Institution and an anonymous donor. All molecular laboratory work was conducted in and with the support of the Laboratories of Analytical Biology facilities of the National Museum of Natural History, Smithsonian Institution. Jennifer Felder assisted in tissue dissection and data-logging during the decapod taxonomy workshop. Arthur Anker identified or confirmed all the Alpheidae material collected during the shrimp workshop. For DLF and JAS, this is contribution number 208 of the UL Laboratory for Crustacean Research.

\section{References}

- $\quad$ Anker A (2001) Two new species of snapping shrimps from the Indo-Pacific, with remarks on colour patterns and sibling species in Alpheidae (Crustacea: Caridea). The Raffles Bulletin of Zoology 49 (1): 57-72.

- $\quad$ Anker A, Hurt C, Knowlton N (2007) Three transisthmian snapping shrimps (Crustacea: Decapoda: Alpheidae: Alpheus) associated with innkeeper worms (Echiura: Thalassematidae) in Panama. Zootaxa 1626 (1): 1-23. https://doi.org/10.11646/zootaxa. 1626.1.1

- $\quad$ Anker A, Hurt C, Knowlton N (2008) Revision of the Alpheus formosus Gibbes, 1850 complex, with redescription of $A$. formosus and description of a new species from the tropical western Atlantic (Crustacea: Decapoda: Alpheidae). Zootaxa 1707: 1-22. https:// doi.org/10.11646/zootaxa.1707.1.1

- $\quad$ Anker A (2010) A new genus and three new species of alpheid shrimps (Crustacea, Decapoda, Caridea) from the tropical American coasts. Zootaxa 2652 (1): 47-63. https:// doi.org/10.11646/zootaxa.2652.1.4

- $\quad$ Anker A, Pachelle PPG, De Grave S, Hultgren KM (2012) Taxonomic and biological notes on some Atlantic species of the snapping shrimp genus Synalpheus Spence Bate, 1888 (Decapoda, Alpheidae). Zootaxa 3598: 1-96.

- Appeltans W, Ahyong S, Anderson G, Angel M, Artois T, Bailly N, Bamber R, Barber A, Bartsch I, Berta A, Błażewicz-Paszkowycz M, Bock P, Boxshall G, Boyko C, Brandão S, Bray R, Bruce N, Cairns S, Chan T, Cheng L, Collins A, Cribb T, Curini-Galletti M, Dahdouh-Guebas F, Davie PF, Dawson M, De Clerck O, Decock W, De Grave S, de Voogd N, Domning D, Emig C, Erséus C, Eschmeyer W, Fauchald K, Fautin D, Feist S, Fransen CJ, Furuya H, Garcia-Alvarez O, Gerken S, Gibson D, Gittenberger A, Gofas S, Gómez-Daglio L, Gordon D, Guiry M, Hernandez F, Hoeksema B, Hopcroft R, Jaume D, Kirk P, Koedam N, Koenemann S, Kolb J, Kristensen R, Kroh A, Lambert G, Lazarus D, Lemaitre R, Longshaw M, Lowry J, Macpherson E, Madin L, Mah C, Mapstone G, McLaughlin P, Mees J, Meland K, Messing C, Mills C, Molodtsova T, Mooi R, Neuhaus B, Ng PL, Nielsen C, Norenburg J, Opresko D, Osawa M, Paulay G, Perrin W, Pilger J, Poore GB, Pugh P, Read G, Reimer J, Rius M, Rocha R, Saiz-Salinas J, Scarabino V, Schierwater B, Schmidt-Rhaesa A, Schnabel K, Schotte M, Schuchert P, Schwabe E, Segers H, Self-Sullivan C, Shenkar N, Siegel V, Sterrer W, Stöhr S, Swalla B, Tasker M, 
Thuesen E, Timm T, Todaro MA, Turon X, Tyler S, Uetz P, van der Land J, Vanhoorne B, van Ofwegen L, van Soest RM, Vanaverbeke J, Walker-Smith G, Walter TC, Warren A, Williams G, Wilson S, Costello M (2012) The magnitude of global marine species diversity. Current Biology 22 (23): 2189-2202. https://doi.org/10.1016/j.cub.2012.09.036

- Bensasson D (2001) Mitochondrial pseudogenes: evolution's misplaced witnesses. Trends in Ecology \& Evolution 16 (6): 314-321. https://doi.org/10.1016/ s0169-5347(01)02151-6

- $\quad$ Bonnet NK, Rocha R (2011) The family Ascidiidae Herdman (Tunicata: Ascidiacea) in Bocas del Toro, Panama. Description of six new species. Zootaxa 2864 (1): 1-33. https://doi.org/10.11646/zootaxa.2864.1.1

- $\quad$ Brasier M, Wiklund H, Neal L, Jeffreys R, Linse K, Ruhl H, Glover A (2016) DNA barcoding uncovers cryptic diversity in $50 \%$ of deep-sea Antarctic polychaetes. Royal Society Open Science 3 (11). https://doi.org/10.1098/rsos.160432

- Bucklin A, Steinke D, Blanco-Bercial L (2011) DNA barcoding of marine metazoa. Annual Review of Marine Science 3 (1): 471-508. https://doi.org/10.1146/annurevmarine-120308-080950

- $\quad$ Bucklin A, Lindeque P, Rodriguez-Ezpeleta N, Albaina A, Lehtiniemi M (2016) Metabarcoding of marine zooplankton: prospects, progress and pitfalls. Journal of Plankton Research 38 (3): 393-400. https://doi.org/10.1093/plankt/fbw023

- Buhay J (2009) "COl-like" sequences are becoming problematic in molecular systematic and DNA barcoding studies. Journal of Crustacean Biology 29 (1): 96-110. https:// doi.org/10.1651/08-3020.1

- Cancian de Araujo B, Schmidt S, Schmidt O, von Rintelen T, Kilmaskossu A, Panjaitan R, Balke M (2018) From field courses to DNA barcoding data release for West Papua making specimens and identifications from university courses more sustainable.

Biodiversity Data Journal 6: e25237. https://doi.org/10.3897/bdj.6.e25237

- $\quad$ Cardoso P, Erwin TL, Borges PAV, New TR (2011) The seven impediments in invertebrate conservation and how to overcome them. Biological Conservation 144 (11): 2647-2655. https://doi.org/10.1016/j.biocon.2011.07.024

- Chak STC, Duffy JE, Hultgren K, Rubenstein D (2017) Evolutionary transitions towards eusociality in snapping shrimps. Nature Ecology \& Evolution 1 (4): 96. https://doi.org/ 10.1038/s41559-017-0096

- Coleman CO (2015) Taxonomy in times of the taxonomic impediment - examples from the community of experts on amphipod crustaceans. Journal of Crustacean Biology 35 (6): 729-740. https://doi.org/10.1163/1937240x-00002381

- Collin R, Díaz MC, Norenburg J, Rocha RM, Sánchez JA, Schulze A, Schwartz M, Valdés A (2005) Photographic identification guide to some common marine invertebrates of Bocas Del Toro, Panama. Caribbean Journal of Science 41 (3): 638-707.

- Collin R, Venera-Pontón D, Driskell A, Macdonald K, Boyle M (2018) Planktotrophic brachiopod larvae from the Pacific and Caribbean of Panama. Diversity 11: 2. https:// doi.org/10.3390/d11010002

- $\quad$ Collin R, Venera-Pontón D, Driskell A, Macdonald K, Chan KK, Boyle M (2019) Documenting neotropical diversity of phoronids with DNAbarcoding of planktonic larvae. Invertebrate Biology 138 (2). https://doi.org/10.1111/ivb.12242

- Costa FO, deWaard JR, Boutillier J, Ratnasingham S, Dooh RT, Hajibabaei M, Hebert PDN (2007) Biological identifications through DNA barcodes: the case of the Crustacea 
. Canadian Journal of Fisheries and Aquatic Sciences 64 (2): 272-295. https://doi.org/ 10.1139/f07-008

- Costello M, Bouchet P, Boxshall G, Fauchald K, Gordon D, Hoeksema B, Poore GB, van Soest RM, Stöhr S, Walter TC, Vanhoorne B, Decock W, Appeltans W (2013) Global coordination and standardisation in marine biodiversity through the World Register of Marine Species (WoRMS) and related databases. PLOS One 8 (1): e51629. https://doi.org/10.1371/journal.pone.0051629

- Cowen R, Gawarkiewicz G, Pineda J, Thorrold S, Werner F (2007) Population connectivity in marine systems: an overview. Oceanography 20 (3): 14-21. https:// doi.org/10.5670/oceanog.2007.26

- $\quad$ Cowen R, Sponaugle S (2009) Larval dispersal and marine population connectivity. Annual Review of Marine Science 1 (1): 443-466. https://doi.org/10.1146/ annurev.marine.010908.163757

- De Grave S, Anker A (2017) An annotated checklist of marine caridean and stenopodidean shrimps (Malacostraca: Decapoda) of the Caribbean coast of Panama. Nauplius 25 https://doi.org/10.1590/2358-2936e2017015

- $\quad$ Drew L (2011) Are we losing the science of taxonomy? BioScience 61 (12): 942-946. https://doi.org/10.1525/bio.2011.61.12.4

- Duffy JE, Morrison C, Ríos R (2000) Multiple origins of eusociality among spongedwelling shrimps (Synalpheus). Evolution 54 (2): 503-516. https://doi.org/10.1111/j. 0014-3820.2000.tb00053.x

- $\quad$ Ebach MC, Valdecasas AG, Wheeler QD (2011) Impediments to taxonomy and users of taxonomy: accessibility and impact evaluation. Cladistics 27 (5): 550-557. https:// doi.org/10.1111/j.1096-0031.2011.00348.x

- $\quad$ Efron B, Halloran E, Holmes S (1996) Bootstrap confidence levels for phylogenetic trees. Proceedings of the National Academy of Sciences 93 (23): 13429-13429. https:// doi.org/10.1073/pnas.93.23.13429

- Felsenstein J (1985) Confidence limits on phylogenies: an approach using the bootstrap. Evolution 39 (4). https://doi.org/10.2307/2408678

- Gascuel O (1997) BIONJ: an improved version of the NJ algorithm based on a simple model of sequence data. Molecular Biology and Evolution 14 (7): 685-695. https:// doi.org/10.1093/oxfordjournals.molbev.a025808

- Geiger M, Moriniere J, Hausmann A, Haszprunar G, Wägele W, Hebert P, Rulik B (2016) Testing the Global Malaise Trap Program - How well does the current barcode reference library identify flying insects in Germany? Biodiversity Data Journal 4: e10671. https://doi.org/10.3897/bdj.4.e10671

- $\quad$ Geller J, Meyer C, Parker M, Hawk H (2013) Redesign of PCR primers for mitochondrial cytochrome $\mathrm{c}$ oxidase subunit I for marine invertebrates and application in all-taxa biotic surveys. Molecular Ecology Resources 13 (5): 851-861. https://doi.org/

10.1111/1755-0998.12138

- $\quad$ Giangrande A (2003) Biodiversity, conservation, and the 'Taxonomic impediment'. Aquatic Conservation: Marine and Freshwater Ecosystems 13 (5): 451-459. https:// doi.org/10.1002/aqc.584

- Goodheart J, Ellingson R, Vital X, Galvão Filho H, McCarthy J, Medrano S, Bhave V, García-Méndez K, Jiménez L, López G, Hoover C, Awbrey J, De Jesus J, Gowacki W, Krug P, Valdés Á (2016) Identification guide to the heterobranch sea slugs (Mollusca: 
Gastropoda) from Bocas del Toro, Panama. Marine Biodiversity Records 9 (1): 56. https://doi.org/10.1186/s41200-016-0048-z

- Hajibabaei M, Singer GC, Hebert PN, Hickey D (2007) DNA barcoding: how it complements taxonomy, molecular phylogenetics and population genetics. Trends in Genetics 23 (4): 167-172. https://doi.org/10.1016/i.tig.2007.02.001

- Harris SE, Bellino M (2013) DNA Barcoding from NYC to Belize. Science 342 (6165): 1462-1463. https://doi.org/10.1126/science.1230006

- Hebert PN, Cywinska A, Ball S, deWaard J (2003) Biological identifications through DNA barcodes. Proceedings of the Royal Society of London. Series B: Biological Sciences 270 (1512): 313-321. https://doi.org/10.1098/rspb.2002.2218

- Hebert PN, Gregory TR (2005) The promise of DNA barcoding for taxonomy. Systematic Biology 54 (5): 852-859. https://doi.org/10.1080/10635150500354886

- Hultgren K, Hurt C, Anker A (2014) Phylogenetic relationships within the snapping shrimp genus Synalpheus (Decapoda: Alpheidae). Molecular Phylogenetics and Evolution 77: 116-125. https://doi.org/10.1016/j.ympev.2014.03.008

- Knowlton N, Weigt LA, Solórzano LA, Mills DK, Bermingham E (1993) Divergence in proteins, mitochondrial DNA, and reproductive compatibility across the isthmus of Panama. Science 260 (5114): 1629-1632. https://doi.org/10.1126/science.8503007

- Kough A, Paris C, Butler M (2013) Larval connectivity and the international management of fisheries. PLOS One 8 (6): e64970. https://doi.org/10.1371/ journal.pone.0064970

- Lassmann T, Sonnhammer E (2005) Kalign--an accurate and fast multiple sequence alignment algorithm. BMC Bioinformatics 6: 298. https://doi.org/

10.1186/1471-2105-6-298

- $\quad$ Leray M, Knowlton N (2015) DNA barcoding and metabarcoding of standardized samples reveal patterns of marine benthic diversity. Proceedings of the National Academy of Sciences 112 (7): 2076-2081. https://doi.org/10.1073/pnas.1424997112

- $\quad$ Liu G, Cui X, Li H, Cai L (2016) Evolutionary direction of processed pseudogenes. Science China Life Sciences 59 (8): 839-849. https://doi.org/10.1007/s11427-016-5074$\underline{x}$

- Mantelatto F, Terossi M, Negri M, Buranelli R, Robles R, Magalhães T, Tamburus AF, Rossi N, Miyazaki M (2017) DNA sequence database as a tool to identify decapod crustaceans on the São Paulo coastline. Mitochondrial DNA Part A 29 (5): 805-815. https://doi.org/10.1080/24701394.2017.1365848

- Mathews L, Anker A (2009) Molecular phylogeny reveals extensive ancient and ongoing radiations in a snapping shrimp species complex (Crustacea, Alpheidae, Alpheus armillatus). Molecular Phylogenetics and Evolution 50 (2): 268-281. https://doi.org/ 10.1016/j.ympev.2008.10.026

- Matzen da Silva J, Creer S, dos Santos A, Costa A, Cunha M, Costa F, Carvalho G (2011) Systematic and evolutionary insights derived from mtDNA COI barcode diversity in the Decapoda (Crustacea: Malacostraca). PLOS One 6 (5): e19449. https://doi.org/ 10.1371/journal.pone.0019449

- Meyer C, Geller J, Paulay G (2005) Fine scale endemism on coral reefs: archipelagic differentiation in turbinid gastropods. Evolution 59 (1): 113-125. https://doi.org/10.1111/j. 0014-3820.2005.tb00899.x 
- $\quad$ Mora C, Tittensor D, Adl S, Simpson AB, Worm B (2011) How many species are there on earth and in the ocean? PLOS Biology 9 (8): e1001127. https://doi.org/10.1371/ journal.pbio. 1001127

- Morín J, Venera-Pontón D, Driskell A, Sánchez J, Lasker H, Collin R (2019) Reference DNA barcodes and other mitochondrial markers for identifying Caribbean octocorals. Biodiversity Data Journal 7 https://doi.org/10.3897/bdj.7.e30970

- Morrison CL, Rios R, Emmett Duffy J (2004) Phylogenetic evidence for an ancient rapid radiation of Caribbean sponge-dwelling snapping shrimps (Synalpheus). Molecular Phylogenetics and Evolution 30 (3): 563-581. https://doi.org/10.1016/ s1055-7903(03)00252-5

- $\quad$ Naro-Maciel E, Reid B, Holmes K, Brumbaugh D, Martin M, DeSalle R (2011) Mitochondrial DNA sequence variation in spiny lobsters: population expansion, panmixia, and divergence. Marine Biology 158 (9): 2027-2041. https://doi.org/10.1007/ s00227-011-1710-y

- $\quad$ Puillandre N, Lambert A, Brouillet S, Achaz G (2011) ABGD, Automatic Barcode Gap Discovery for primary species delimitation. Molecular Ecology 21 (8): 1864-1877. https://doi.org/10.1111/j.1365-294x.2011.05239.x

- Ratnasingham S, Hebert PDN (2007) BOLD: the barcode of life data system (http:// www.barcodinglife.org). Molecular Ecology Notes 7 (3): 355-364. https://doi.org/10.1111/ j.1471-8286.2007.01678.x

- Ratnasingham S, Hebert PDN (2013) A DNA-based registry for all animal species: the barcode index number (BIN) system. PLOS One 8 (7): e66213. https://doi.org/10.1371/ journal.pone.0066213

- $\quad$ Raupach M, Radulovici A (2015) Looking back on a decade of barcoding crustaceans. ZooKeys 539: 53-81. https://doi.org/10.3897/zookeys.539.6530

- Raupach M, Barco A, Steinke D, Beermann J, Laakmann S, Mohrbeck I, Neumann H, Kihara T, Pointner K, Radulovici A, Segelken-Voigt A, Wesse C, Knebelsberger T (2015) The application of DNA barcodes for the identification of marine crustaceans from the North Sea and adjacent regions. PLOS One 10 (9): e139421. https://doi.org/10.1371/ journal.pone. 0139421

- Rocha RM, Faria SB, Moreno TR (2005) Ascidians from Bocas del Toro, Panama. I. Biodiversity. Caribbean Journal of Science 41 (3): 600-612.

- Schill S, Raber G, Roberts J, Treml E, Brenner J, Halpin P (2015) No reef is an island: integrating coral reef connectivity data into the design of regional-scale marine protected area networks. PLOS One 10 (12): e144199. https://doi.org/10.1371/ journal.pone.0144199

- Schizas NV (2012) Misconceptions regarding nuclear mitochondrial pseudogenes (Numts) may obscure detection of mitochondrial evolutionary novelties. Aquatic Biology 17 (1): 91-96. https://doi.org/10.3354/ab00478

- Schneider-Broussard R, Neigel JE (1997) A large-subunit mitochondrial ribosomal DNA sequence translocated to the nuclear genome of two stone crabs (Menippe). Molecular Biology and Evolution 14 (2): 156-165. https://doi.org/10.1093/ oxfordjournals.molbev.a025748

- Schubart CD (2010) Mitochondrial DNA and decapod phylogenies: the importance of pseudogenes and primer optimization. Decapod Crustacean Phylogenetics. Crustacean Issues 18: 47-65. https://doi.org/10.1201/9781420092592-c4 
- Sluys R (2013) The unappreciated, fundamentally analytical nature of taxonomy and the implications for the inventory of biodiversity. Biodiversity and Conservation 22 (4):

1095-1105. https://doi.org/10.1007/s10531-013-0472-x

- $\quad$ Song H, Buhay JE, Whiting MF, Crandall KA (2008) Many species in one: DNA barcoding overestimates the number of species when nuclear mitochondrial pseudogenes are coamplified. Proceedings of the National Academy of Sciences 105 (36): 13486-13491. https://doi.org/10.1073/pnas.0803076105

- Vences M, Thomas M, van der Meijden A, Chiari Y, Vieites DR (2005) Comparative performance of the $16 \mathrm{~S}$ rRNA gene in DNA barcoding of amphibians. Frontiers in zoology 2 (1): 5. https://doi.org/10.1186/1742-9994-2-5

- Vernooy R, Haribabu E, Muller MR, Vogel JH, Hebert PDN, Schindel DE, Shimura J, Singer GAC (2010) Barcoding life to conserve biological diversity: beyond the taxonomic imperative. PLOS Biology 8 (7): e1000417. https://doi.org/10.1371/ journal.pbio. 1000417

- Wägele H, Klussmann-Kolb A, Kuhlmann M, Haszprunar G, Lindberg D, Koch A, Wägele JW (2011) The taxonomist - an endangered race. A practical proposal for its survival. Frontiers in Zoology 8 (1). https://doi.org/10.1186/1742-9994-8-25

- Williams ST, Knowlton N (2001) Mitochondrial pseudogenes are pervasive and often insidious in the snapping shrimp genus Alpheus . Molecular Biology and Evolution 18 (8): 1484-1493. https://doi.org/10.1093/oxfordjournals.molbev.a003934

- $\quad$ Zheng L, He J, Lin Y, Cao W, Zhang W (2014) 16S rRNA is a better choice than COI for DNA barcoding hydrozoans in the coastal waters of China. Acta Oceanologica Sinica 33 (4): 55-76. https://doi.org/10.1007/s13131-014-0415-8 